\title{
Interplanetary magnetic field control of Saturn's polar cusp aurora
}

\author{
E. J. Bunce, S. W. H. Cowley, and S. E. Milan \\ Department of Physics and Astronomy, University of Leicester, Leicester LE1 7RH, UK \\ Received: 21 September 2004 - Revised: 3 February 2005 - Accepted: 8 February 2005 - Published: 3 June 2005
}

\begin{abstract}
Dayside UV emissions in Saturn's polar ionosphere have been suggested to be the first observational evidence of the kronian "cusp" (Gérard et al., 2004). The emission has two distinct states. The first is a bright arclike feature located in the pre-noon sector, and the second is a more diffuse "spot" of aurora which lies poleward of the general location of the main auroral oval, which may be related to different upstream interplanetary magnetic field (IMF) orientations. Here we take up the suggestion that these emissions correspond to the cusp. However, direct precipitation of electrons in the cusp regions is not capable of producing significant UV aurora. We have therefore investigated the possibility that the observed UV emissions are associated with reconnection occurring at the dayside magnetopause, possibly pulsed, akin to flux transfer events seen at the Earth. We devise a conceptual model of pulsed reconnection at the low-latitude dayside magnetopause for the case of northwards IMF which will give rise to pulsed twinvortical flows in the magnetosphere and ionosphere in the vicinity of the open-closed field-line boundary, and hence to bi-polar field-aligned currents centred in the vortical flows. During intervals of high-latitude lobe reconnection for southward IMF, we also expect to have pulsed twin-vortical flows and corresponding bi-polar field-aligned currents. The vortical flows in this case, however, are displaced poleward of the open-closed field line boundary, and are reversed in sense, such that the field-aligned currents are also reversed. For both cases of northward and southward IMF we have also for the first time included the effects associated with the IMF $B_{y}$ effect. We also include the modulation introduced by the structured nature of the solar wind and IMF at Saturn's orbit by developing "slow" and "fast" flow models corresponding to intermediate and high strength IMF respectively. We then consider the conditions under which the plasma populations appropriate to either sub-solar reconnection or highlatitude lobe reconnection can carry the currents indicated. We have estimated the field-aligned voltages required, the resulting precipitating particle energy fluxes, and the consequent auroral output. Overall our model of pulsed reconnection under conditions of northwards and southwards IMF,
\end{abstract}

Correspondence to: E. J. Bunce

(emma.bunce@ion.le.ac.uk) and for varying orientations of IMF $B_{y}$, is found to produce a range of UV emission intensities and geometries which is in good agreement with the data presented by Gerrard et al. (2004). The recent HST-Cassini solar wind campaign provides a unique opportunity to test the theoretical ideas presented here.

Keywords. Magnetospheric physics (Auroral phenomena; Planetary magnetospheres; Solar wind-magnetosphere interactions)

\section{Introduction}

The high-latitude far-ultraviolet (FUV) aurora in Saturn's ionosphere has been observed by a variety of spacecraft and telescopes. Originally, it was data from the Pioneer-11 spacecraft that indicated the existence of the UV emissions (Judge et al., 1980), together with remotely sensed observations from the IUE spacecraft (Clarke et al., 1981). Subsequently, the UVS instruments on board Voyager-1 and -2 clarified these earlier measurements (Broadfoot et al., 1981; Sandel and Broadfoot, 1981; Sandel et al., 1982; Shemansky and Ajello, 1983). However, more recently, increasing resolution and sensitivity by the Hubble Space Telescope (HST) has become available (Gérard et al., 1995, 2004; Trauger et al., 1998; Cowley et al., 2004). These latter observations have enabled some general auroral characteristics to be established. The FUV emission is due to $\mathrm{keV}$ electron impact on Saturn's hydrogen atmosphere and generally takes the form of a narrow ring of aurora at $\sim 15^{\circ}$ co-latitude. Auroral intensities vary between $\sim 1 \mathrm{kR}$ and $\sim 100 \mathrm{kR}$, peaking in the pre-noon sector and are often stronger at dawn than at dusk. Recently, Stallard et al. (2004) have made Doppler measurements of the infrared (IR) auroras at Saturn, and have shown that the polar ionosphere significantly subcorotates relative to the planet, with an angular velocity of $\sim 20-40 \%$ of rigid corotation. In January 2004, the HST made observations of the UV aurora whilst the Cassini spacecraft sampled the in-situ solar wind plasma and magnetic field and remotely observed the radio emissions from Saturn's polar regions. These coupled data sets will inevitably increase our understanding of the morphology and origin of Saturn's aurora, 
as the details become available. In general these data have revealed the substantial variability in the morphology, colatitude and intensity of the emission (Clarke et al., 2004) which is suggested to be related to structure in the solar wind and interplanetary magnetic field (e.g. Jackman et al., 2004; Clarke et al., 2004, Cowley et al., 2004, 2005).

Concurrent with observations, theoretical work has centred on the origins of the auroras at Saturn, and their implications for magnetospheric dynamics. Due to the "discrete" nature of the aurora it is generally accepted that the UV emissions are associated with regions of upward field-aligned currents (i.e. downgoing electrons). Essentially, there are two basic sources of such large-scale field-aligned currents which transfer momentum and energy in the solar windmagnetosphere-ionosphere system. The first is that which is associated with the transfer of angular momentum from the atmosphere of the rapidly rotating planet to the subcorotating equatorial plasma (Hill, 1979; Vasyliunas, 1983). At Jupiter, the angular momentum transfer is required by the radial outflow of sulphur and oxygen plasma from the Io torus, and it is this large-scale system of field-aligned currents which has been suggested to be driving the main auroral oval emissions in Jupiter's ionosphere (Bunce and Cowley, 2001; Cowley and Bunce, 2001; Hill, 2001; Southwood and Kivelson, 2001). At Saturn, the plasma source from the icy moons and ring grains is thought to be approximately an order of magnitude smaller than that at Jupiter (see, for example, Richardson 1992; Richardson et al., 1998; Saur et al., 2004). Voyager observations of the plasma angular velocity indicate that despite this difference the radial outflow of plasma at Saturn is sufficient to cause the breakdown of corotation (Richardson, 1986, 1995; Richardson and Sittler, 1990), and hence the generation of the aforementioned largescale magnetosphere-ionosphere coupling current system. In a recent paper, however, Cowley and Bunce (2003) have considered the properties of this current system at Saturn, by employing simple models of the equatorial plasma angular velocity and the magnetic field (both of which are based on Voyager data), together with an estimate of the ionospheric conductivity made by Bunce et al. (2003). In summary, the results suggest that the region of upward field-aligned current associated with magnetosphere-ionosphere coupling is both too weak in magnitude and flow at too large a co-latitude $\left(\sim 20^{\circ}\right.$ co-latitude) to reasonably account for the implied auroral currents associated with Saturn's main auroral oval.

The second possibility is that the aurora is related to those currents which are driven by the interaction between the solar wind, and its embedded interplanetary magnetic field, with that of the planet. This process functions principally via magnetic reconnection at the magnetopause and the Dungey cycle (Dungey, 1961). This external solar wind actuation is the main source of auroral field-aligned currents at Earth (see, e.g. Paschmann et al., 2002, and references therein). Following on from the work of Cowley and Bunce (2003), Cowley et al. (2004) have suggested that Saturn's auroras are associated with a ring of upward current along the open-closed field line boundary generated by the difference in angular velocity of open and outer closed field lines (see Sect. 2 for a more detailed discussion). However, these currents will also be significantly modulated by the solar wind and interplanetary magnetic field, as at Earth.

Recently, Gérard et al. (2004) have published all of the pre-January 2004 HST data for Saturn and have systematically discussed the features present. Interestingly, they find evidence for a dayside emission observed near noon, which they suggest is the first observational evidence for Saturn's polar cusp. The dayside emission they observe exists in two states, a morning arc-like emission lying along the main auroral oval, and a high-latitude "spot" of more diffuse aurora. Two examples of the morphology of Saturn's southern UV aurora is projected into the ionosphere and shown in Figs. 1a and b, taken from Gérard et al. (2004). In Fig. 1a we see the main auroral oval which is brighter at dawn than at dusk (as predicted by Cowley et al., 2004), with a brightening along the oval in the pre-noon sector. In Fig. $1 \mathrm{~b}$ we can see the high-latitude "spot" poleward of the main oval location. The intensity of these two latter emissions ranges between 8-70 kR for the "morning arc", and 10-20 kR for the highlatitude "spot". This bi-modal state, Gérard et al. (2004) suggest, could be indicative of differing states of the interplanetary magnetic field (IMF), as is the case at Earth (Milan et al., 2000). At Jupiter, it has also been suggested that a particular feature of the dayside polar emissions is related to the dayside cusp (Pallier and Prangé, 2001; Waite et al., 2001; Grodent et al., 2003). This consists of a patch of bright UV emission seen in both the Northern and Southern Hemispheres (Pallier and Prangé, 2004) and is highly variable in intensity. The spatial extent of this feature is large, a few $1000 \mathrm{~km}$, and is consistently located poleward of the main auroral oval. The UV dayside emission appears to be related to both the polar "flare" emission reported by Waite et al. (2001), and also to the main source of jovian X-rays (e.g. Metzger et al., 1983; Waite et al., 1994) suggested by the Chandra X-ray Observatory (CXO) to be originating in a polar "hotspot" which appears to be pulsing with $\sim 45$ min periodicity (Gladstone et al., 2002). Motivated by the pulsed nature of the X-rays, Bunce et al. (2004) have recently devised a conceptual model of the twin vortical flows and bi-polar currents which straddle the open-closed field line boundary during intervals of pulsed reconnection at the dayside magnetopause for the simple case of sub-solar reconnection during northward IMF (i.e. $B_{z}>0$ ). Depending on the details of the model, the results indicate that emissions associated with dayside reconnection vary over a few tens of $k R$ to a few tens of MR, in keeping with the reported levels of emission. Bunce et al. (2004) also suggest that MV acceleration of magnetospheric oxygen in the downward current region can also provide a significant source of X-ray emission, at the level of a few GW, in good agreement with the observations.

Here we investigate the possibility of a cusp aurora at Saturn associated with pulsed reconnection at the dayside magnetopause, following on from the model devised for Jupiter by Bunce et al. (2004). The context of our discussion begins 
with a more detailed consideration of the large-scale flows and currents in Saturn's polar ionosphere than that discussed recently by Cowley et al. (2004). We then extend these steady-state flows to include the localised effects of transient flows and currents associated with transient dayside reconnection. Motivated by the known dependence of the Earth's cusp emissions on the upstream IMF orientation, we also extend the model to include the effects of varying IMF $B_{z}$ and $B_{y}$ orientation, and compare the results with the observations of Gérard et al. (2004). We specifically consider the implications of the model flows and currents for UV emission at Saturn from precipitating magnetosheath and magnetospheric particle populations with regard to our model of sub-solar reconnection during northwards IMF, and from the lobe magnetosheath and plasma mantle appropriate for southwards IMF and high-latitude reconnection. We will focus on regions of upward-directed field-aligned currents, and their consequences, as it is these currents which are mainly responsible for the aurora at Earth.

\section{Background}

An appropriate framework for the consideration of dayside cusp processes is shown in Fig. 1c, taken from Cowley et al. (2004), which was briefly discussed in the Introduction. This picture depicts the flows and currents in Saturn's polar regions, and is based on the consideration of three main physical regimes discussed previously by Hill (1979), Vasyliunas (1983), and Dungey (1961). These regimes are shown in Fig. 1c in a view which is looking down onto the northern ionosphere, with the pole at the centre, in a frame which is fixed relative to the Sun. The solid lines indicate plasma streamlines, whilst the circled dots and crosses indicate regions of field-aligned current directed upward and downward respectively. The three flow regions are as follows Eq. 1 a lower-latitude region which maps to the sub-corotating plasma in the equatorial plane, associated with the fieldaligned current system which transfers momentum from the ionosphere to the magnetosphere, discussed above, which is of insufficient magnitude and flowing at the wrong colatitude to account for the main oval auroras at Saturn (Cowley and Bunce, 2003), Eq. 2 a higher-latitude region of subcorotating flows where field lines are stretched out downtail and eventually pinch off, forming a plasmoid, which is subsequently released downtail (the "Vasyliunas cycle"), and Eq. 3 a region of flow which is driven by reconnection at the dayside magnetopause in which "open" field lines mapping to the tail lobes flow anti-sunward over the poles, and following reconnection in the tail, return to the dayside, drawn here principally via dawn, in a single cell convection pattern (the "Dungey cycle"). The single cell convection pattern leads to the conjecture that the main "steady-state" oval will be stronger at dawn than at dusk, which is also evident in Fig. 1a. It is the effects associated with the "Dungeycycle magnetopause X-line" shown in Fig. 1c which will be
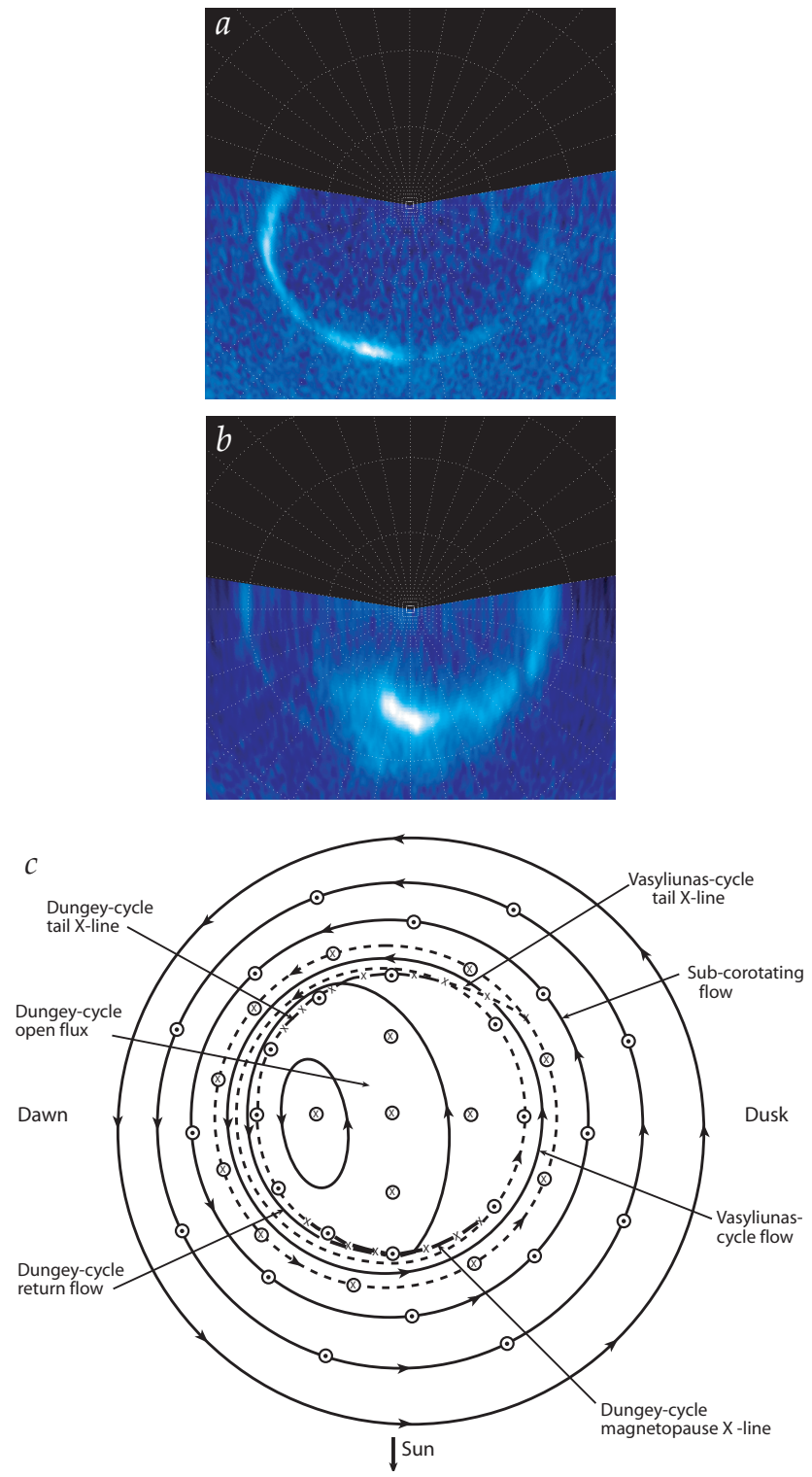

Fig. 1. Two examples of the morphology of Saturn's southern aurora obtained with the HST-STIS $\mathrm{SrF}_{2}$ filter. Images are projected onto the ionosphere, where the pole is to the centre, and circles of increasing size indicate $80^{\circ}, 70^{\circ}$, and $60^{\circ}$ of latitude respectively. The direction to the Sun is at the bottom of the diagram, dusk is to the right and dawn to the left. Longitude meridians are shown at intervals of $10^{\circ}$. Image (a), taken on the 29th January 2001, shows an auroral oval which is brighter at dawn than at dusk, with an additional brightening in the pre-noon sector. Image (b) shows the high-latitude "spot" discussed in the text. (From Gérard et al., 2004). (c) Sketch of the flows and field-aligned currents in Saturn's ionosphere, in the same view as Fig. 1a. Arrowed solid lines show plasma streamlines and arrowed short-dashed lines show the boundaries between flow regimes (also streamlines). Long-dashed lines marked with Xs indicate the ionospheric mapping of the reconnection site (X-lines) associated with the Dungey and Vasyliunas cycles. Circled dots and crosses indicate regions of upward and downward field-aligned current respectively. (From Cowley et al., 2004). 
pertinent to this study, i.e. where reconnection takes place between planetary and magnetosheath magnetic field lines.

Observations of the Earth's ionospheric flow configuration show that there are three main effects on the flow structure, which depend on the upstream IMF orientation (e.g. Cowley and Lockwood, 1992). The first is the large-scale two-cell flow pattern, with anti-sunward flows at high-latitudes over the polar cap and return sunward flows at lower latitudes. This scenario corresponds to solar wind-driven convection when the IMF is directed southwards at Earth i.e. $B_{z}<0$, and is in keeping with the "open" model of the magnetosphere suggested by Dungey (1961). Of course this corresponds to the northward IMF case at Jupiter and Saturn due to the oppositely direct planetary fields. Under these conditions magnetic flux is added to the polar cap which causes the boundary to expand. The second regime consists of sunward-directed flows which are observed in the central polar cap region. Flows of this nature are typically observed when the IMF has a significant positive $B_{z}$ (Maezawa, 1976; Burke et al., 1979; Heelis et al., 1986). This flow pattern is thought to be produced by high-latitude reconnection between the IMF and pre-existing open flux tubes in the tail lobes. In the most likely scenario whereby the IMF reconnects with one lobe only, the amount of open flux in the system remains constant. Thirdly, the flows described above during intervals of both southward and northward directed IMF orientations also exhibit dawn-dusk asymmetries, which are oppositely directed in the Northern and Southern Hemispheres, and whose sense depends on the east-west component $\left(B_{y}\right)$ of the IMF (Jørgensen et al., 1972; Cowley, 1981). This asymmetry is explained in theoretical terms as simply being the effect of the east-west tension force which is exerted on newly-opened field lines in the presence of IMF $B_{y}$. As before, the sense of this asymmetry is opposite for the cases of Jupiter and Saturn to that which is observed at the Earth in a given hemisphere. These various flow regimes have been discussed in terms of localised flows and currents in the Earth's cusp region, where each of these IMF conditions subsequently result in varying cusp auroral activity (Farrugia et al., 1995; Øieroset et al., 1997; Sandholt et al., 1998; Milan et al., 2000).

In the case of Saturn, reconnection at the sub-solar dayside magnetopause between magnetosheath and magnetospheric field lines, leads to the transfer of magnetic flux across the open-closed field line boundary (labelled the Dungey cycle reconnection X-line in Fig. 1c) under conditions of northward IMF. The "active" portion of this boundary where reconnection is ongoing at the magnetopause at any one time, mapping to the magnetopause reconnection $\mathrm{X}$-line, will be termed the "merging gap" in this discussion which is in keeping with terrestrial terminology (e.g. Lockwood et al., 1990). In Fig. 1c, this merging gap stretches over a few hours of local time, where steady reconnection is driving this largescale convection cell associated with the Dungey cycle (during northward IMF) as discussed above. However, if dayside reconnection is transient or pulsed, as observed at Earth in "flux transfer events" (FTEs) (Russell and Elphic, 1978; Provan et al., 1998; Neudegg et al., 1999; Wild et al., 2001), then the reconnection may occur across a more restricted sector of the boundary than is shown here. Under these circumstances, the reconnection pulses give rise to more localised flow features across this boundary than has been depicted in Fig. 1c. As discussed by Bunce et al. (2004), for the case of northwards IMF we would expect transient poleward flows across the merging gap, as newly opened field lines are transferred across the open-closed field line boundary from the dayside magnetopause across the polar cap, and a slower transient equatorward flow on either side, as the boundary of open field lines expands equatorward. In general then, such pulses of reconnection will create twin-vortex flows in the vicinity of the open-closed field line boundary (e.g. Siscoe and Huang, 1985; Cowley and Lockwood, 1992), as described above and which we will model in Sect. 3 using the same form of the flows and currents induced by pulsed reconnection at Jupiter, presented by Bunce et al. (2004). In Fig. 2a we show a simple schematic depicting the sense of the currents and flows for the case of sub-solar reconnection discussed above. Here we also attempt to model the transient effects on the flows and currents produced during intervals of high-latitude lobe reconnection which occur during intervals of southward IMF, an effect which is known to produce corresponding cusp aurora at Earth (Farrugia et al, 1995; Øieroset et al., 1997; Sandholt et al., 1998; Milan et al., 2000; Phan et al., 2004). During intervals of southward IMF, reconnection takes place at high-latitudes and the merging gap is thus displaced poleward of the open-closed field line boundary. Flows associated with high-latitude reconnection at the Earth indicate that twin-vortical flows are still present, but that the direction of flow is reversed and confined to the polar cap, in keeping with the above discussion (Maezawa, 1976; Burke et al., 1979; Heelis et al, 1986). The reversed high-latitude currents and flows associated with southward IMF are sketched in Fig. 2b. In addition, we have considered the asymmetrical ionospheric flow effects which will be introduced by IMF $B_{y}$.

Newly-opened magnetic field lines lying just poleward of the merging gap in the case of sub-solar reconnection during northwards IMF, map from the ionosphere through the magnetosphere to the magnetopause and form the magnetic structure of the magnetospheric "cusp" region. Plasma crossing the magnetopause onto magnetospheric field lines is then expected to precipitate into the ionosphere just poleward of the merging gap, forming a "cusp plasma" precipitation region, similar to that which is observed at the Earth (e.g. Reiff et al., 1980). In addition, magnetospheric plasma from the outer magnetosphere at lower latitudes will also precipitate and form part of the "cusp plasma". In the case of southwards IMF, the newly-reconnected high-latitude magnetic field lines are located equatorward of the merging gap as the plasma flows sunward, and the "cusp plasma" crossing the magnetopause is that of the cooler, more tenuous lobe magnetosheath and also that of the plasma mantle. An important point to recognise at this juncture is that this precipitation without acceleration cannot be directly responsible for any substantial auroral emission at Saturn, as was also found to 
be the case at Jupiter (Bunce et al. (2004). If we first consider the precipitation of sub-solar magnetosheath electrons, with typical densities of $0.02-0.2 \mathrm{~cm}^{-3}$ and temperatures $\sim 50 \mathrm{eV}$ (Sittler et al., 1983; Richardson and Sittler, 1990; Richardson, 1995), we find that the precipitating energy flux lies between $\sim 0.4$ and $4.0 \mu \mathrm{W} \mathrm{m}^{-2}$, which at $10-20 \%$ conversion efficiency into $\sim 10 \mathrm{eV} \mathrm{UV}$ photons is sufficient to produce a UV aurora of only $\sim 0.004$ to $0.04 \mathrm{kR}$, which is orders of magnitude below the dayside arc and high-latitude emissions reported by Gérard et al. (2004). We may also consider direct precipitation of electrons from the outer magnetosphere as candidates for producing auroral emission. For example, the outer magnetospheric hot electron component with typical densities of $0.01 \mathrm{~cm}^{-3}$ and temperatures of $\sim 600 \mathrm{eV}$ (Sittler et al., 1983; Richardson and Sittler, 1990; Richardson, 1995) produce a precipitating energy flux of $\sim 17 \mu \mathrm{W} \mathrm{m}^{-2}$ which would indicate an auroral UV output of $\sim 0.17 \mathrm{kR}$, which is again much less than the reported UV intensities. In the high-latitude reconnection regime we consider electrons precipitating from the high-latitude magnetosheath and also from the region known as the "plasma mantle" at Earth (i.e. that region of the high-latitude lobe containing plasma originating from the magnetosheath). In the high-latitude magnetosheath we may expect, by analogy with the Earth, to find typical densities and temperatures which are $\sim 1 / 2$ those found in the sub-solar magnetosheath (e.g. Spreiter et al., 1966). Therefore with densities of $\sim 0.1-0.01 \mathrm{~cm}^{-3}$ and temperatures of $\sim 25 \mathrm{eV}$, we find that the energy flux ranges between $0.0067-0.067 \mu \mathrm{W} \mathrm{m}^{-2}$, with again negligible UV emission. Similarly, by comparison with the Earth, electrons in the plasma mantle will typically have a density of a tenth of those found in the high-latitude magnetosheath, while having comparable temperatures to those in the lobe magnetosheath (see Sckopke and Paschmann, 1978). Again, these populations produce between 0.067 and $0.67 \mu \mathrm{W} \mathrm{m}^{-2}$ and hence no observable UV emissions.

The estimates of the energy flux of precipitating electrons and the subsequent UV emission from the regions discussed above show the necessity for substantial acceleration of magnetospheric, plasma mantle and/or magnetosheath particles in order to account for the observed emission intensities. In view of this, we will make a related study to that discussed by Bunce et al. (2004) for Jupiter, considering first the flows and currents likely to be associated with both sub-solar and high-latitude reconnection at the dayside magnetopause, for various orientations of IMF $B_{y}$. Subsequently, we first consider the particle acceleration required in regions of upwarddirected field-aligned current during intervals of sub-solar reconnection and second during occurrences of high-latitude lobe reconnection, and estimate the level of auroral emission present. Regions of downward-directed field-aligned current will be assumed to be carried by upgoing electrons rather than downgoing ions, which will be discussed in more detail in Sect. 4.

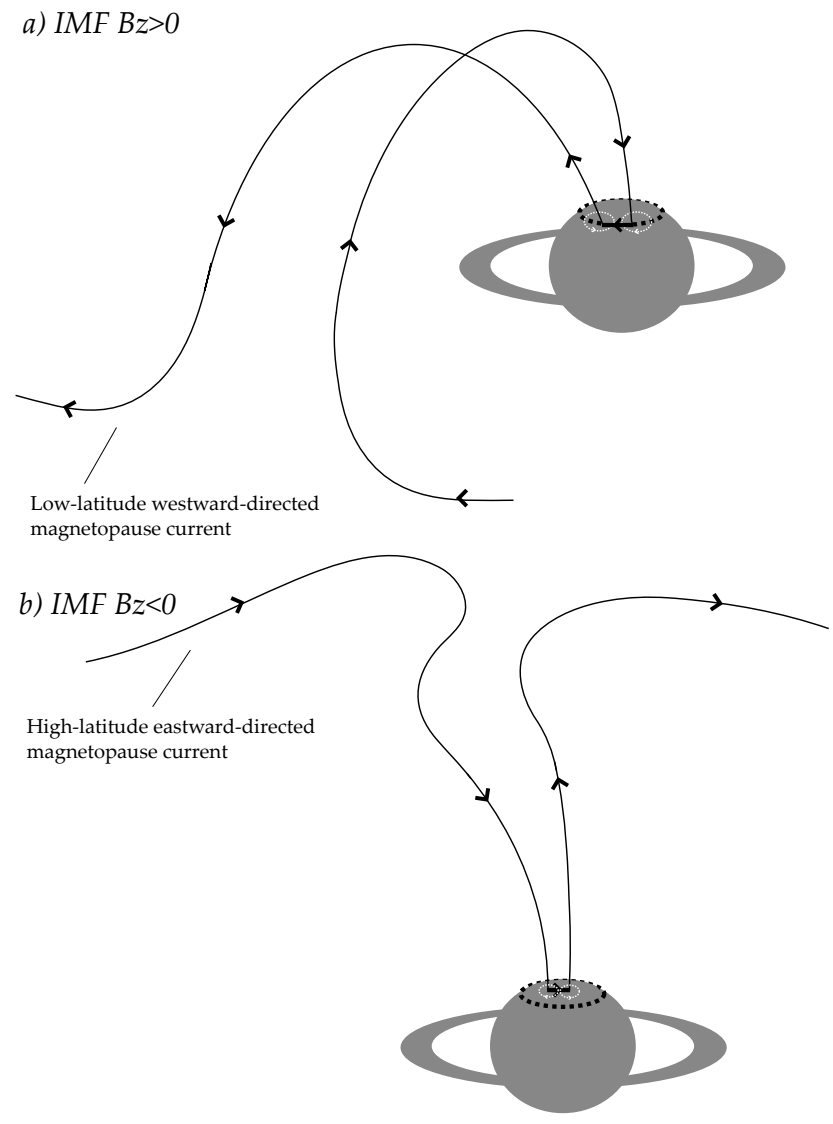

Fig. 2. Schematic indicating the sense of the large-scale currents (solid black lines) which flow during (a) sub-solar and (b) highlatitude reconnection for intervals of northwards and southwards IMF respectively. In sketch a) the low-latitude westward-directed field-perpendicular magnetopause currents are diverted downwards into the ionosphere at the eastern end of the merging gap, and upwards at the western end of the merging gap. The currents close in this case via westward-directed Pedersen currents in the ionosphere. This current system is responsible for transferring momentum from the magnetopause to the ionosphere, driving the vortical flow pattern depicted by the dashed white lines. In sketch b) the high-latitude eastward-directed magnetopause currents are diverted downwards into the ionosphere at the western end of the merging gap, and upwards at the eastern end of the merging gap. The currents close in this case via eastward-directed Pedersen currents in the ionosphere thus reversing the sense of the vortical flows depicted again by the dashed white lines. In both cases, the dashed black line in the ionosphere depicts the open-closed field line boundary.

\section{Simple models of the flows and currents in Saturn's ionosphere due to pulsed reconnection at the dayside magnetopause under varying IMF orientations}

Recently, Jackman et al. (2004) have investigated the reconnection-driven interaction of the solar wind with Saturn's magnetosphere with particular focus on the consequences for magnetospheric dynamics. IMF data obtained by the Cassini spacecraft en route to Saturn was collected for 8 complete solar rotations which allow the variation of 
the field structure to be investigated. They find that, as at Jupiter, the solar wind magnetic field structure is consistent with that expected to be produced by corotating interaction regions (CIRs) during the declining phase of the solar cycle, when the Sun's dipole field is tilted at $\sim 30^{\circ}$ with respect to the spin axis. In general the data show that currently the IMF structure consists of two sectors during each rotation of the Sun, with crossings of the heliospheric current sheet generally embedded within few-day high field compression regions, surrounded by several day low-field rarefaction regions.

Such upstream IMF data can be employed to directly estimate the dayside reconnection voltage across Saturn's magnetopause associated with the production of open flux, and cusp related phenomena, based on analogy with the results of studies made at the Earth. The structuring of the IMF into regions of high and low field strength is mirrored in the estimate of the reconnection voltage, and hence in the interaction with the magnetosphere. The estimate for the dayside "driving" voltage is given by

$\Phi=V_{s w} B_{\perp} L_{0} \cos ^{4}\left(\frac{\theta}{2}\right)$,

where $V_{s w}$ is the radial speed of the solar wind, $B_{\perp}$ is the strength of the IMF component perpendicular to the radial flow, $L_{0}$ is a scale length taken to equal $10 \mathrm{R}_{s}$ (where $\left.1 \mathrm{R}_{s}=60330 \mathrm{~km}\right)$ by analogy with the Earth, and $\theta$ is the clock-angle of the IMF relative to Saturn's northern magnetic axis. According to Eq. (1), large dayside reconnection voltages are favoured for northward pointing IMF at Saturn, opposite to the case for Earth due to the opposite sense of the planetary field. Here we use the results of Jackman et al. (2004) to estimate the transpolar voltage generated by the solar wind interaction. During rarefaction regions, the IMF typically has two distinct regimes of field strength. We observe extended intervals of weak IMF where the field strength is very low ( $\sim 0.1 \mathrm{nT}$ or less), such that the estimated voltages are $\sim 10 \mathrm{kV}$ or less. Jackman et al. (2004) comment on the "essential absence of magnetopause reconnection" during such low field strength intervals. Secondly, there are intervals when the IMF is of "intermediate" strength, i.e. the magnetic field strength is $\sim 0.2-0.8 \mathrm{nT}$. The estimated reconnection voltage produced during such episodes is highly variable due to north-south field variations, but on average is $\sim 50 \mathrm{kV}$, and may have peaks of $\sim 150 \mathrm{kV}$. The high-field strength compression regions are relatively much shorter (i.e. few days) than the several day rarefaction regions (Jackman et al., 2004). The field strength during these "active" times varies generally in the range $\sim 0.5-2.0 \mathrm{nT}$, which inevitably produces much larger reconnection voltages. The average value is typically $\sim 110 \mathrm{kV}$, with up to $\sim 400 \mathrm{kV}$ at the peaks.

As discussed in the previous section, transient processes such as dayside reconnection, while occurring over some reasonable portion of the dayside magnetopause, may generally map to a more restricted sector of the boundary than has been sketched in Fig. 1c, which is intended to represent an overall steady-state picture. The open-closed field line boundary depicted in Fig. 1c is taken to lie at $\sim 15^{\circ}$ dipole co-latitude (based on available auroral information), with the dayside merging gap (or mapped magnetopause $\mathrm{X}$ line) extending over longitudinal distances in the ionosphere of $\sim 20000 \mathrm{~km}$ (i.e. $\sim 5 \mathrm{~h}$ of local time). However, for the case of time-dependent reconnection that we are considering here, it seems reasonable to suppose that the instantaneous merging gap may be more restricted longitudinally than this, and hence we consider a merging gap of $\sim 5000 \mathrm{~km}$ length (i.e. $\sim 1 \mathrm{~h}$ of local time), in keeping with the discussion by Bunce et al. (2004). Since this width is much smaller than that of the whole open-flux region sketched in Fig. 1c, the flow speeds in the vicinity of the merging gap will be correspondingly higher than those generally present in the polar cap for the same voltage as quoted above. The same is true for the high-latitude merging gap present during intervals of southward IMF. The flow speeds in the vicinity of the merging gap may also be enhanced by the characteristic pulsing of the reconnection process at the magnetopause. At Earth the typical duration between reconnection pulses (i.e. between FTEs) is $\sim 8 \mathrm{~min}$ (e.g. McWilliams et al., 2000). This interval roughly corresponds to the Alfvén wave transit time from one hemisphere to the other and back again along the outer dayside field lines. Bunce et al. (2004) have taken this suggestion and estimated an analogous time-scale for Jupiter to be $\sim 30-50 \mathrm{~min}$ (which supports the possibility that the $\mathrm{X}$-rays at Jupiter may be associated with bursts of reconnection). At Saturn an estimate may be made of this time-scale from the field-strength and plasma density in the outer magnetosphere using the simple dipole field formulation of Cummings (1969). For a magnetic field strength of $\sim 8 \mathrm{nT}$ in the outer magnetosphere, and a density of $\sim 0.01 \mathrm{~cm}^{-3}$, we find that the Alfvén wave transit time is $\sim 20-30$ min for Saturn's magnetosphere. We, therefore, suggest that we may observe some characteristic periodicities of $\sim 20-30 \mathrm{~min}$ in various parameters such as electron beams and radio emissions at Saturn, as have been seen at Jupiter.

Based upon the discussion above, we then have chosen two model regimes. For sub-solar reconnection during intervals of northward IMF, we have first modelled the intermediate IMF conditions using a transpolar voltage of $\sim 100 \mathrm{kV}$ corresponding to a transpolar flow speed on open field lines (across a polar cap of $\sim 15^{\circ}$ ) of $\sim 70 \mathrm{~m} \mathrm{~s}^{-1}$. This model is intended to represent reconnection voltages which are larger than the average under such conditions $(\sim 50 \mathrm{kV})$, and somewhat less than the peaks $(150 \mathrm{kV})$ observed during the intermediate IMF strength regime. This model is our "slow" flow model (in keeping with the discussion by Bunce et al. (2004) in the context of Jupiter). We do not model the low IMF $(\sim 0.1 \mathrm{nT})$, which produces $\sim 10 \mathrm{kV}$ or less, and is suggested to produce no significant dayside reconnection (Jackman et al., 2004). Conversely, in the "fast" model we take a transpolar voltage of $\sim 400 \mathrm{kV}$, closest to the peaks in the strongfield IMF compression regions, which implies a transpolar flow speed of $\sim 300 \mathrm{~m} \mathrm{~s}^{-1}$. For lobe reconnection under conditions of southward IMF, we have taken the potential to be a half of that during intervals of northwards IMF. This is based 
upon the terrestrial study of Milan (2004), which will be discussed in more detail below. The values of the potential chosen here are intended to represent the flows due to pulsed reconnection at some reasonably developed stage, and we recognise that at the peak of the pulsed flow the values may be somewhat increased from those discussed here. However, it is important to note that the same flows will be present (perhaps on a larger scale) during steady reconnection such that the results discussed here do not necessarily require that reconnection be pulsed.

We have therefore developed a simple model of the ionospheric flows and currents which are likely to exist in the vicinity of the merging gap during intervals of pulsed reconnection. The flow and hence current geometry are designed to be analogous to those which have been observed at Earth under various different IMF orientations for northward or southward IMF $B_{z}$ and for positive and negative IMF $B_{y}$ in each case. For simplicity we consider a locally plane (Cartesian) coordinate system, in which $y$ points north, whilst $x$ is positive east. The line $y=0$ is taken to be the instantaneous location of the open-closed field line boundary. The ionospheric magnetic field $\mathbf{B}_{i}=B_{i} \hat{\mathbf{z}}$ is assumed for simplicity to be uniform and vertical, and we have also assumed that the height-integrated ionospheric conductivity is uniform. As discussed by Bunce et al. (2004), the basic equations are

$\mathbf{E}_{i}=-\nabla \Phi_{i}=-\mathbf{V}_{i} \wedge \mathbf{B}_{i}$,

where $E_{i}$ is the ionospheric electric field, $\Phi_{i}$ the electrostatic potential (the electric field being curl-free), and $V_{i}$ is the ionospheric plasma velocity,

$\mathbf{i}=\mathbf{i}_{P}+\mathbf{i}_{H}=\Sigma_{P} \mathbf{E}_{i}^{\prime}+\Sigma_{H} \hat{\mathbf{B}}_{i} \wedge \mathbf{E}_{i}^{\prime}$

where $i$ is the height-integrated ionospheric current intensity, consisting of Pedersen, $i_{P}$, and Hall, $i_{H}$, components, $\Sigma_{P}$ and $\Sigma_{H}$ are the height-integrated Pedersen and Hall conductances respectively, and $\mathbf{E}_{i}^{\prime}$ is the electric field in the neutral atmosphere rest frame, given by

$\mathbf{E}_{i}^{\prime}=-\left(\mathbf{V}_{i}-\mathbf{V}_{n}\right) \wedge \mathbf{B}_{i}$,

where $V_{n}$ is the velocity of the neutral atmosphere, and finally

$j_{\| i}=-\operatorname{divi}$

where $j_{\| i}$ is the field-aligned current density, positive when directed along the field lines out of the ionosphere. The neutral atmosphere is assumed to be essentially rigidly corotating with the planet, and thus moving uniformly eastward (i.e. in the $\mathrm{x}$-direction) with a speed, at $\sim 15^{\circ}$ co-latitude, of $\sim 2.6 \mathrm{~km} \mathrm{~s}^{-1}$ in the inertial frame. In order to consider the auroral emissions which may be present associated with the reconnection process, we must consider the field-aligned current density, and the subsequent conditions in the magnetospheric and magnetosheath plasma which are required to carry them. Thus substituting Eqs. (3) and (4) into (5), and assuming that $V_{n}, \Sigma_{P}$, and $\Sigma_{H}$ are all constants, as indicated above, yields

$j_{\| i}=-\operatorname{div} \mathbf{i}_{P}=\Sigma_{P} \mathbf{B}_{i} \cdot \operatorname{curl} \mathbf{V}_{i}=\Sigma_{P} \nabla^{2} \Phi_{i}$.
The field-aligned current density is therefore simply derived from specification of the velocity field, which is equivalent to the ionospheric electrostatic potential, since electrostatic potentials represent streamlines of the flow. Here we first specify a "background" flow, on top of which a twin-vortical flow pattern has been superposed, representing the instantaneous effect of pulsed reconnection under either northwards or southwards IMF orientation. We will also include the effects of IMF $B_{y}$, which will be discussed in more detail below. For the background flow we have taken, as a simple convenient form

$$
\begin{aligned}
& \Phi_{1}(x, y)=B_{i} \\
& {\left[V_{1 y} x+\frac{\left(V_{1 x}-V_{2 x}-V_{3 x}\right) y_{0}}{2}\left[\log \cosh \left(\frac{y}{y_{0}}\right)-\left(V_{1 x}+V_{2 x}+V_{3 x}\right)\left(\frac{y}{y_{0}}\right)\right]\right],}
\end{aligned}
$$

such that

$$
\begin{aligned}
& \mathbf{V}_{1}(x, y)= \\
& {\left[\frac{1}{2}\left(\left(V_{1 x}+V_{2 x}+V_{3 x}\right)-\left(V_{1 x}-V_{2 x}-V_{3 x}\right) \tanh \left(\frac{y}{y_{0}}\right)\right) \hat{\mathbf{x}}+V_{1 y} \hat{\mathbf{y}}\right],}
\end{aligned}
$$

and

$j_{\| i 1}=\frac{\Sigma_{P} B_{i}\left(V_{1 x}-V_{2 x}-V_{3 x}\right)}{2 y_{0}} \frac{1}{\cosh ^{2}\left(\frac{y}{y_{0}}\right)}$.

The flow is therefore such that the eastward $(x)$ component changes from an essentially uniform flow at speed $V_{1 x}$ for $y<0$, representing closed field lines, to $V_{2 x}+V_{3 x}$ for $y>0$, representing open field lines (we recall from above that the instantaneous open-closed field line boundary is taken to lie along the line $y=0$ ). With reference to Fig. $1, V_{1 x}$ thus represents the sub-corotational transport in the Dungey cycle "return" flow and Vasyliunas-cycle flow equatorward of the open-closed field line boundary. In accordance with Cowley et al. (2004), we have assumed this flow to take place at a speed which is a reasonable fraction of rigid corotation ( $\sim 2.6 \mathrm{~km} \mathrm{~s}^{-1}$ as above), such that here we have taken it to be $\sim 80 \%$ of this value i.e. $V_{1 x}=2.0 \mathrm{~km} \mathrm{~s}^{-1}$. In addition, as observed by Stallard et al. (2004), and considered theoretically by Cowley et al. (2004), there is also a slow rotation of the plasma in the open-field region at $\sim 30 \%$ of rigid co-rotation, i.e. $V_{2 x}=0.75 \mathrm{~km} \mathrm{~s}^{-1}$. Finally, $V_{3 x}$ represents a steady-state IMF $B_{y}$ related flow which is simply directed eastward or westward for the case of positive or negative IMF $B_{y}$ respectively. We choose reasonably small values of $100 \mathrm{~m} \mathrm{~s}^{-1}$ for the high strength IMF model, and $10 \mathrm{~m} \mathrm{~s}^{-1}$ for the intermediate strength IMF model. The transition from eastward speed $V_{1 x}$ on closed field lines to $V_{2 x}+V_{3 x}$ on open field lines then takes place on spatial scale $y_{0}$ across the open-closed field line boundary, a distance we have taken somewhat arbitrarily to be equal to $500 \mathrm{~km}$. Thus the eastward flow transition of the background flow is assumed to take place on latitudinal scales of $\sim 1^{\circ}$. With these values, an ionospheric height-integrated Pedersen conductivity of $1.0 \mathrm{mho}$, as assumed throughout here, representing a value within the range 
derived by Bunce et al. (2003), and $B_{i}=6.5 \times 10^{-5} \mathrm{~T}$, the peak value of the field-aligned current density at $y=0$ due to the flow shear is then found from Eq. (7c) to be $0.16 \mu \mathrm{Am}^{-2}$. The uniform northward flow in Eq. (7b) at speed $V_{1 y}$ then represents the steady flow poleward from the dayside to the nightside associated with the Dungey cycle. In conformity with the above discussion, we have taken this to be $70 \mathrm{~m} \mathrm{~s}^{-1}$ during intermediate strength IMF $B_{z}$ northward intervals, and $300 \mathrm{~m} \mathrm{~s}^{-1}$ during high field strength IMF $B_{z}$ northward intervals. In the case of southward IMF $B_{z}$ this steady-state anti-sunward flow is set to zero representing the cessation of dayside magnetospheric driving for this magnetic field geometry. That is to say that no magnetic flux transfer takes place across the merging gap for this IMF orientation (see for example Cowley and Lockwood, 1992).

3.1 Vortical flows due to pulsed reconnection for northward or southward IMF $B_{z}$ and $B_{y}=0$

We first describe the flows due to pulsed reconnection at the dayside magnetopause for the two simple cases of northward or southward IMF $B_{z}$ during intervals when IMF $B_{y}$ is zero by the addition of vortical flows superimposed on the background flows described in the previous section. For the case of northwards IMF these instantaneous flows are modelled as being twin vortex in form, consisting of the sum of two oppositely directed vortices, centred near the two ends of the merging gap along the open-closed field line boundary for the case of northwards IMF. For the case of southwards IMF, similar flows are employed, but the twin vortex and merging gap is displaced to higher latitudes poleward of the openclosed field line boundary, and the sense of rotation is reversed. This is similar to the conditions which exist in the Earth's magnetosphere for the case of high-latitude "lobe" reconnection (Cowley and Lockwood, 1992; Øieroset et al., 1997; Taylor et al., 1998). When $B_{y}=0$ we take the simple forms

$$
\begin{aligned}
& \Phi_{2 \pm}(x, y) \\
& = \pm B_{i} V_{2} \rho_{0} \log \left[1+\frac{\left(\left(x \pm x_{0}\right)^{2}+r_{2} y^{2}\right)}{\rho_{0}^{2}}\right]
\end{aligned}
$$

such that the streamlines of each individual vortex are elliptical, and where the upper sign corresponds to the counterclockwise vortex centred near the western end of the merging gap, while the lower sign corresponds to the clockwise vortex centred near the eastern end of the merging gap,

$$
\begin{aligned}
& \mathbf{V}_{2_{ \pm}}(x, y)= \\
& \frac{ \pm 2 V_{2}}{\left(1+\frac{\left(\left(x \pm x_{0}\right)^{2}+r^{2} y^{2}\right)}{\rho_{0}^{2}}\right)}\left[-r^{2}\left(\frac{y}{\rho_{0}}\right) \hat{\mathbf{x}}+\left(\frac{x \pm x_{0}}{\rho_{0}}\right) \hat{\mathbf{y}}\right]
\end{aligned}
$$

and

$j_{\| i} \pm(x, y)=$

$$
\pm \frac{2 \Sigma_{P} B_{i} V_{2}}{\rho_{0}} \frac{\left[\left(r^{2}+1\right)+\left(r^{2}-1\right)\left[\left(\frac{x \pm x_{0}}{\rho_{0}}\right)^{2}-r^{2}\left(\frac{y}{\rho_{0}}\right)^{2}\right]\right]}{\left[1+\frac{\left(\left(x \pm x_{0}\right)^{2}+r^{2} y^{2}\right)}{\rho_{0}^{2}}\right]^{2} .}
$$

The total flow associated with the reconnection pulse is then taken as the sum of the positive and negative flow contributions. These add together to form a twin-vortex pattern as mentioned previously, with a strong poleward flow across the merging gap, representing the flow driven by the transfer of field lines across the open-closed field line boundary (i.e. $y=0$ ) from the dayside magnetosphere into the tail, reversing to a weaker equatorward flow on the boundary on either side, representing the consequent expansion of the region of open field lines.

With regard to the values of the parameters in Eq. (8), we have chosen $x_{0}=2300 \mathrm{~km}$ and $\rho_{0}=800 \mathrm{~km}$, such that the north-south flow rapidly reverses sense at the ends of the merging gap at $x= \pm 2500 \mathrm{~km}$ (giving an overall merging gap length of $5000 \mathrm{~km}$ as indicated above), and is relatively constant in the region between. The value of $V_{2}$ has then been chosen such that the total voltage along the merging gap associated with the flow is $\sim 100 \mathrm{kV}$ in the case of the intermediate strength IMF model, and $\sim 400 \mathrm{kV}$ in the high strength IMF model, also in conformity with the above discussion. The corresponding values of $V_{2}$ are $0.175 \mathrm{~km} \mathrm{~s}^{-1}$ in the first case and $0.70 \mathrm{~km} \mathrm{~s}^{-1}$ in the second. The northward flow at the centre of the merging gap $(x=y=0)$ due to the two vortices combined is then $\sim 0.25 \mathrm{~km} \mathrm{~s}^{-1}$ in the first case, and $\sim 0.90 \mathrm{~km} \mathrm{~s}^{-1}$ in the second. For convenience, we shall henceforth refer to the intermediate IMF case as the "slow" flow model, and the high strength IMF case as the "fast" flow model. The value of $r$ (representing the ratio of the major to minor axes of the elliptical streamlines) has been taken to be 2.5 , in order to represent the spread of the flow streamlines to east and west away from the merging gap along the open-closed field line boundary. The peak fieldaligned current density at the centre of each flow vortex is then found to be approximately $\pm 0.21 \mu \mathrm{A} \mathrm{m}^{-2}$ for the slow flow model, and $\pm 0.83 \mu \mathrm{A} \mathrm{m}^{-2}$ for the fast flow model, with upward currents flowing in the anti-clockwise vortex centred at the western end of the merging gap, and downward currents flowing in the clockwise vortex centred at the eastern end. We note that these peak field-aligned current densities are greater than that associated with the background flow (which peaks at $\sim 0.16 \mu \mathrm{A} \mathrm{m}^{-2}$ along the open-closed field line boundary as noted above) by a factor only of $\sim 1.3$ in the former case, but by a factor of $\sim 5.2$ in the latter case.

We use the same format of the vortical flows discussed above also for the case of southwards IMF $B_{z}$. Under these conditions, we simply move the merging gap poleward of the open-closed field line boundary as discussed above by a distance which is somewhat arbitrarily chosen to be $2000 \mathrm{~km}$, and reverse the sense of the flow vortices. In terms of Eq. (8), the upper sign now corresponds to the clockwise vortex centred near the western end of the merging gap, while the lower sign corresponds to the counter-clockwise vortex centred 
near the eastern end of the merging gap. The twin-vortical flows for the case of southwards IMF $B_{z}$ now add together to form a total flow which is directed sunward near noon, due to the field tension force on the magnetic flux tubes under the high-latitude reconnection field geometry. The subsequent anti-sunward flows on either side then represent the effect of the magnetosheath flow dragging the flux tubes into the tail (e.g. Cowley and Lockwood, 1992). With regard to the parameters in Eq. (8) we continue to choose $x_{0}=2300 \mathrm{~km}$, $\rho_{0}=800 \mathrm{~km}$, and $r=2.5$, for reasons outlined above. We justify our choice of the potential available from the solar wind by analogy with recent terrestrial studies (Milan, 2004; Milan et al. 2004). These studies indicate that the cross polar cap potential for the case of northwards IMF (at Earth) is some fraction of the southward IMF case. For simplicity here, therefore, we take the potential for the case of southwards IMF (at Saturn) to be approximately half that in the northwards IMF model. The value of $V_{2}$ has then been chosen such that the total voltage along the merging gap associated with the flow is $\sim 50 \mathrm{kV}$ for the slow flow model and $\sim 200 \mathrm{kV}$ for the fast flow model. The flows are also reduced and the corresponding values of $V_{2}$ are $0.087 \mathrm{~km} \mathrm{~s}^{-1}$ in the first case and $0.35 \mathrm{~km} \mathrm{~s}^{-1}$ in the second. The southward flow at the centre of the merging gap $(x=y=0)$ due to the two vortices combined is then $\sim 0.11 \mathrm{~km} \mathrm{~s}^{-1}$ in the first case, and $\sim 0.45 \mathrm{~km} \mathrm{~s}^{-1}$ in the second. The peak field-aligned current density at the centre of each flow vortex is also found to be approximately $\pm 0.11 \mu \mathrm{A} \mathrm{m}^{-2}$ for the slow flow model, and $\pm 0.41 \mu \mathrm{A} \mathrm{m}^{-2}$ for the fast flow model, with upward currents flowing in the anti-clockwise vortex centred at the eastern end of the merging gap, and downward currents flowing in the clockwise vortex centred at the western end opposite to the case for $B_{z}$ northwards. These peak field-aligned current densities are comparable to that associated with the background flow (i.e. $\sim 0.16 \mu \mathrm{A} \mathrm{m}^{-2}$ along the open-closed field line boundary as noted previously) for the case of the slow flow model, but a factor of $\sim 2.5$ larger in the fast flow model case.

Maps showing the streamlines of the flow, equivalently the electric equipotentials (left column), and contours of the field-aligned current density (right column) are shown for both the fast and slow flow models in Fig. 3. The top two rows show results for the fast flow model, whilst the bottom two rows show results for the slow flow model (as indicated to the right of the panels). In the left column, streamlines are shown at intervals of $50 \mathrm{kV}$ in each case. The top row and third row show model conditions for northward IMF $B_{z}$, while the second and fourth rows show model conditions for southward IMF $B_{z}$. As indicated above, it should be emphasized that these models are intended to represent surges of flow across the corresponding merging gaps due to a reconnection pulse, at a point in the evolution of the flow which is reasonably developed. Following the reconnection pulse on the magnetopause, the flow will grow to that illustrated here over a $\sim 10$ min interval, after which it will decline again to the background over several tens of minutes.

The right hand column depicts field-aligned current den- sity contours corresponding to the flows shown in the left hand panel. The contours are labelled 0, 0.05, 0.1, 0.25, 0.5, 1.0 for the different models in units of $\mu \mathrm{A} \mathrm{m}^{-2}$. We recall here that it has been suggested that the background current is responsible for the main oval emissions at Saturn (Cowley et al., 2004), and hence pulsed reconnection for the case of $B_{z}>0$ represents an instantaneous perturbation of these field-aligned currents, enhancing the upwarddirected current (and hence aurora) in the pre-noon sector, whilst reducing or reversing the field-aligned current in the post-noon sector. For the case of the fast and slow flow model and IMF $B_{z}>0$, the contours show how the upwarddirected field-aligned current density along the open-closed field boundary associated with the background flows results in the restriction of the downward field-aligned current density associated with the vortical flows to a smaller region. The upward-directed field aligned current associated with the vortical flows due to pulsed reconnection, in both the fast and slow flow models, is then enhanced by this background current. The peak upward-directed current in the prenoon sector is $\sim 1.0 \mu \mathrm{A} \mathrm{m}^{-2}$ for the fast flow model, and $0.37 \mu \mathrm{A} \mathrm{m}^{-2}$ for the slow model. Similarly, in the post-noon sector, the downward-directed field-aligned current peaks at $-0.64 \mu \mathrm{A} \mathrm{m}^{-2}$ for the fast flow model, and $-0.04 \mu \mathrm{A} \mathrm{m}^{-2}$ for the slow flow model. The extent to which the additional bi-polar field-aligned current pair superposed on the background "main oval" field-aligned current modifies the UV auroral output will be discussed in the next Section.

For the case of $B_{z}>0$, we also note that the main fieldaligned currents associated with the vortical flows, upward at the western end of the merging gap, and downward at the eastern end, are flanked to the north and south by oppositelydirected field-aligned currents of weaker intensity. These currents are associated with the "channeling" of the flow along the boundary due to the assumed elliptical streamlines, described by the parameter $r$, and disappear for circular vortices with $r=1$. In effect these currents represent vestiges within the model of the "region 2" and "cusp" currents as observed at Earth on closed and open field lines, respectively, while the main currents correspond to "region 1" currents. However, the former field-aligned currents are weaker than the latter, hence we do not discuss them further here, but concentrate only on the main field-aligned currents centred on the flow vortices.

For the case of $B_{z}<0$, the vortical flows and resulting bipolar field-aligned current pair (which is directed upwards at the eastern end of the merging gap) are shifted to higher latitude. For the fast model, the field-aligned current peaks are $\pm 0.40 \mu \mathrm{A} \mathrm{m}^{-2}$, whilst in the slow model the peaks values are much less at $\pm 0.11 \mu \mathrm{A} \mathrm{m}^{-2}$. In fact, the contours show that the upward-directed field-aligned current density in the boundary region associated with the background flow is essentially unaffected by the vortical flows associated with a pulse of high-latitude reconnection.

It is of interest to consider not only the field-aligned current densities as above, but also the total currents flowing in the bi-polar regions. For the case of northwards IMF 


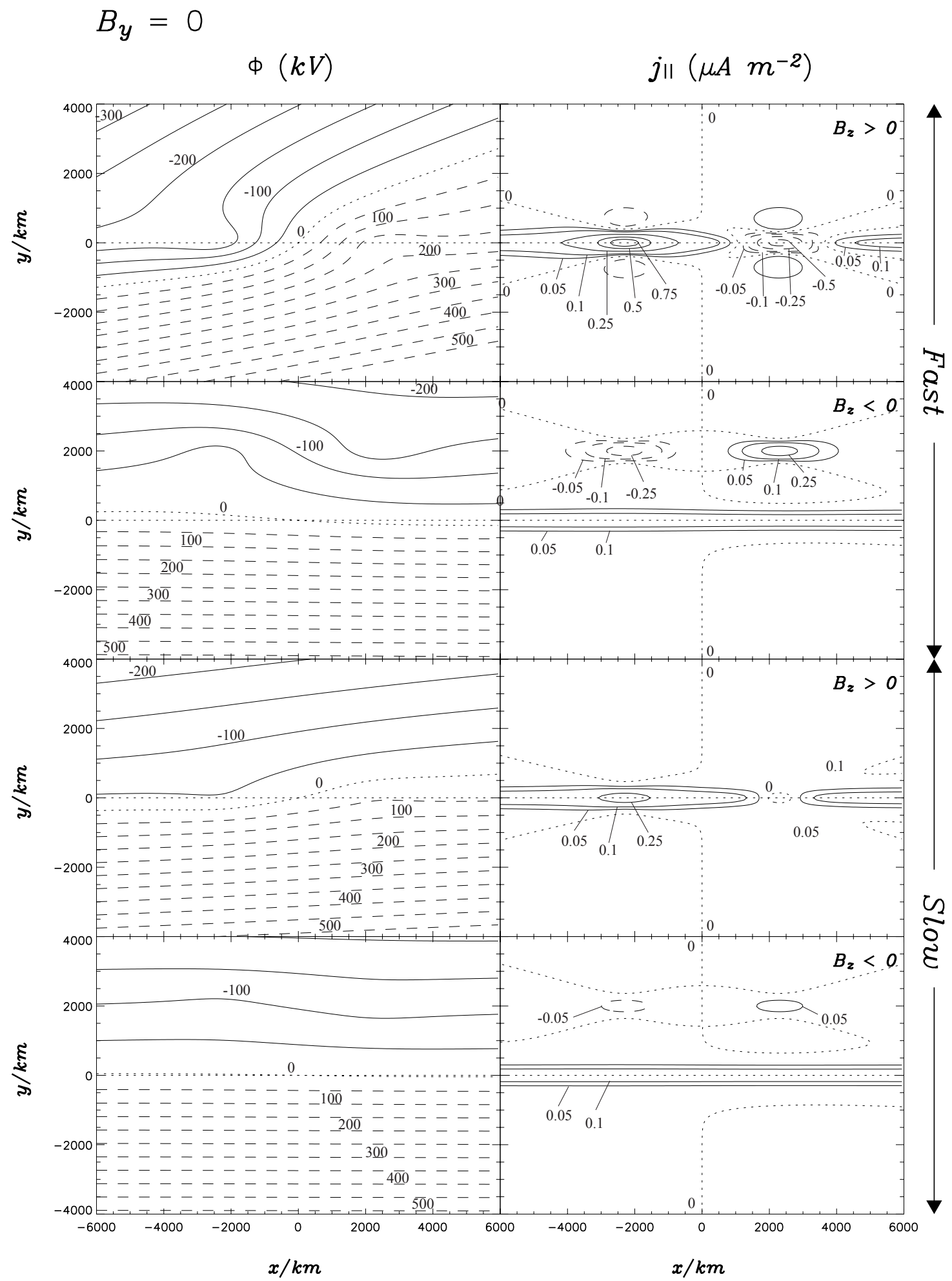

Fig. 3. Here we show a stack plot of electrostatic potential contours (i.e. streamlines of the plasma flow) on the left, and field-aligned current density on the right, for the case of our "fast flow" model in the first two rows, and for the "slow flow" model in the second two rows. The first row in each case corresponds to the case of $B_{z}>0$, and the second to the case of $B_{z}<0$ as shown. Both are presented on equivalent grids of $-4000-4000 \mathrm{~km}$ in $y$, and $-6000-6000 \mathrm{~km}$ in $x$. The short-dashed line at $y=0$ indicates the open-closed field line boundary. On the left, in the plasma streamlines grid the dashed lines show contours of negative electrostatic potential. Contours are labelled in steps of $50 \mathrm{kV}$. On the right, the field-aligned current grids show dotted lines indicating contours of zero field-aligned current density. Solid lines indicate the regions of upward-directed field-aligned current density while the dashed lines indicate the regions of downward-directed field-aligned current density. Contours are labelled 0.05, 0.1, 0.25, 0.5, 0.75, and 1.0, in units of $\mu \mathrm{A} \mathrm{m}^{2}$. 
$B_{z}$, the total upward and downward current flowing e.g. in the rectangles from -6000 to $0 \mathrm{~km}$ in $x$ and $\pm 350 \mathrm{~km}$ in $y$, are $\pm 0.60 \mathrm{MA}$ for the fast flow model and $\pm 0.15 \mathrm{MA}$ for the slow model. These currents will eventually flow to the magnetopause, where they form a "magnetopause current wedge". This implies that the westward flowing ChapmanFerraro current on the magnetopause (as sketched in Fig. 2a) is diverted down the field lines into the eastern vortex, flows across the ionosphere as a Pedersen current driven by the vortical flow pattern, and subsequently flows back up the field lines to the magnetopause out of the western vortex. This process thus weakens the westward flowing ChapmanFerraro magnetopause current during the reconnection interval. The total magnitude of the field-aligned currents associated with the vortices corresponds to currents which are $<10 \%$ of the total Chapman-Ferraro magnetopause current flowing in each hemisphere. Inclusion of the background field-aligned currents, however, increases the total upward field-aligned current near the western vortex from $+0.60 \mathrm{MA}$ to +1.02 MA for the fast model in the same area as above, while the total downward current above the eastern vortex is restricted to a region between 1500 to $3250 \mathrm{~km}$ in $x$ and $\pm 350 \mathrm{~km}$ in $y$, where the current is then reduced from $-0.60 \mathrm{MA}$ to $-0.25 \mathrm{MA}$. For the slow flow model, the upward current in the western rectangle increases from 0.15 MA to 0.58 MA when the background currents are included, whilst the downward current region is much reduced to an area of 2000 to $2500 \mathrm{~km}$ in $x$ and $\pm 200 \mathrm{~km}$ in $y$, with a total downward current reduced to just $\sim-0.01 \mathrm{MA}$.

In the case when the IMF is southwards, we consider the total currents flowing in the rectangle -3500 to $-1000 \mathrm{~km}$ in $x$, and 1750 to 2250 in $y$ for the downward current at the western end of the merging gap, and from $1000 \mathrm{~km}$ to $3500 \mathrm{~km}$ in $x$, and 1750 to 2250 in $y$ for the upward current at the eastern end of the merging gap. The total currents flowing in the reversed high-latitude vortices alone are $\pm 0.21 \mathrm{MA}$ for the fast model. For the slow model, over the same area, the total currents are $\pm 0.05 \mathrm{MA}$. The currents estimated here for the case $B_{z}<0$ eventually flow to the high-latitude magnetopause, where they form a similar "magnetopause current wedge" configuration as discussed above for the case of northwards IMF. The Chapman-Ferraro magnetopause current, which flows eastward at high-latitudes due to the opposite field orientation (sketched in Fig. 2b), is therefore equivalently weakened in the region between the reversed highlatitude vortices representative of southward IMF conditions. We note that the magnitudes of the total field-aligned currents involved represent currents which are also $\leq 10 \%$ of the total Chapman-Ferraro magnetopause currents flowing in each lobe.

\subsection{Flow and current effects associated with IMF $B_{y}$}

Here, we now show how the model of the flows and currents described above for the two simple cases of IMF $B_{z}$ positive and negative, can be modified to include effects associated with positive and negative orientations of the IMF $B_{y}$ com- ponent. For simplicity we employ the same form of flow as described above for the case $B_{y}=0$, but now introduce an asymmetry in the size, strength, and ellipticity of the vortices representing the eastward or westward flows due to the tension force associated with IMF $B_{y}$. Here we thus generalise Eq. (8) to use:

$$
\begin{aligned}
& \Phi_{2 \pm}(x, y)= \pm B_{i} V_{2 \pm} \rho_{0 \pm} \log \left[1+\frac{\left(\left(x \pm x_{0}\right)^{2}+r_{ \pm}^{2} y^{2}\right)}{\rho_{0 \pm}^{2}}\right] \\
& \mathbf{V}_{2_{ \pm}}(x, y)=\frac{ \pm 2 V_{2 \pm}}{\left(1+\frac{\left(\left(x \pm x_{0}\right)^{2}+r_{ \pm}^{2} y^{2}\right)}{\rho_{0 \pm}^{2}}\right)} \\
& {\left[-r_{ \pm}^{2}\left(\frac{y}{\rho_{0 \pm}}\right) \hat{\mathbf{x}}+\left(\frac{x \pm x_{0}}{\rho_{0 \pm}}\right) \hat{\mathbf{y}}\right]}
\end{aligned}
$$

and

$$
\begin{aligned}
& j_{\| i} \pm(x, y)= \\
& \pm \frac{2 \Sigma_{P} B_{i} V_{2 \pm}}{\rho_{0 \pm}} \frac{\left[\left(r_{ \pm}^{2}+1\right)+\left(r_{ \pm}^{2}-1\right)\left[\left(\frac{x \pm x_{0}}{\rho_{0 \pm}}\right)^{2}-r_{ \pm}^{2}\left(\frac{y}{\rho_{0 \pm}}\right)^{2}\right]\right]}{\left[1+\frac{\left(\left(x \pm x_{0}\right)^{2}+r_{ \pm}^{2} y^{2}\right)}{\rho_{0 \pm}^{2}}\right]^{2}} .
\end{aligned}
$$

where $V_{2 \pm}=V_{2} \times f_{ \pm}$modifies the strength of the ellipse, whilst $\rho_{0 \pm}=\frac{800}{f_{ \pm}}$changes its size. The upper sign corresponds again to the flow vortex at the western end of the merging gap, and the lower sign to the flow vortex at the eastern end of the merging gap. In this formulation, the product $V_{2 \pm} \rho_{0 \pm}$ remains constant, regardless of the asymmetry introduced in the vortical flows. Similarly, the value $r$ indicating the ellipticity of the flow vortex takes an upper or lower sign for the western and eastern vortex respectively. To represent IMF $B_{y}>0$ (independent of IMF $B_{z}$ ) we take $f_{+}=0.8$ in the western vortex and $f_{-}=1.2$ in the eastern vortex, whilst $r_{+}=1.0$ in the western vortex and $r_{-}=2.5$ in the eastern vortex. The result of this is to decrease the ellipticity of the western vortex, and also to decrease its strength and size. The eastern vortex thus has a larger strength, size and ellipticity in comparison, and hence the general flow both poleward and equatorward of the merging gap is directed eastward. To represent IMF $B_{y}<0$, we take $f_{+}=1.2$ in the western vortex and $f_{-}=0.8$ in the eastern vortex, whilst $r_{+}=2.5$ in the western vortex and $r_{-}=1.0$ in the eastern vortex. The result of this is to decrease the ellipticity of the eastern vortex, and also to decrease its strength and size. The western vortex has a larger strength, size and ellipticity in comparison, and hence the general plasma flow both poleward and equatorward of the merging gap is directed westward. In Fig. 4 we show electrostatic potential contours (or equivalently plasma flow streamlines) for the vortical flows alone. In each case the solid lines indicate anti-clockwise vortices (hence regions of upwarddirected field-aligned currents) and the dashed lines indicate clockwise vortices (hence regions of upward-directed fieldaligned currents). In each row we show vortical flows for northward IMF on the left, and southward IMF on the right. 


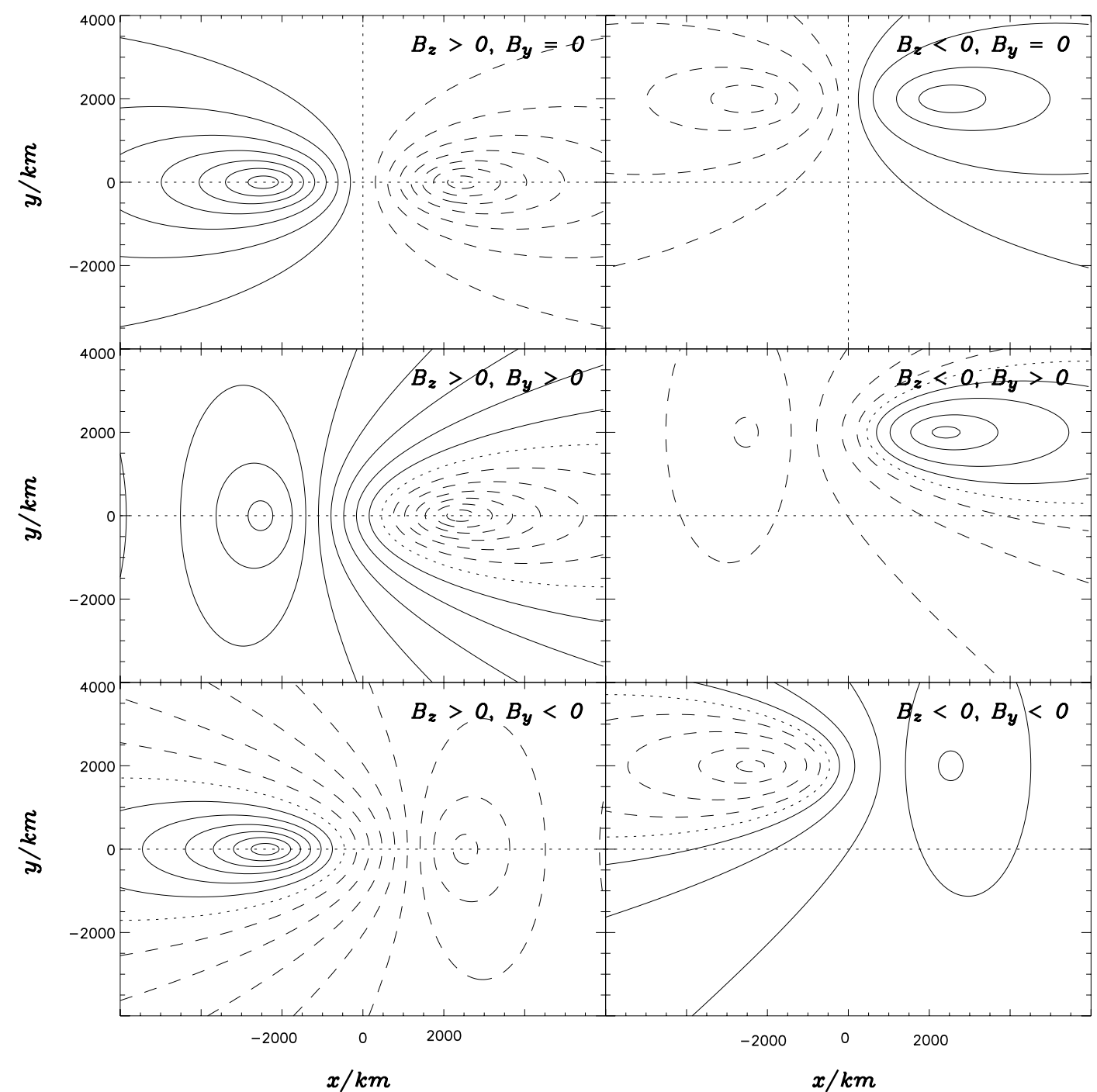

Fig. 4. Here we show a stack plot of electrostatic potential contours (i.e. streamlines of the plasma flow) due to the vortical flows alone, for northward IMF on the left and southward IMF on the right. The three rows correspond to the case of $B_{y}=0, B_{y}>0$, and $B_{y}<0$ respectively, as indicated. The solid lines indicate anti-clockwise vortices, whilst the dashed lines indicate clockwise vortices. The dotted line indicates the line of zero potential. Each case is shown on the same grid as Fig. 3.

The case of $B_{y}=0$ is shown in the top row. The effect of changing the vortices as discussed above is then readily seen in the second and third rows, for IMF $B_{y}>0$ and $B_{y}<0$. We recognise that the streamlines should not cross the openclosed field line boundary $(y=0)$, but note that as the flows are small here anyway, and in order to retain computational simplicity, we leave the model as it stands.

In Figs. 5 and 6 maps showing the streamlines of the flow, equivalently the electric equipotentials (left column), and contours of the field-aligned current density (right column) are shown for both the fast and slow flow models in the same format as in Fig. 3. As discussed above, the flows are intended to represent a time which is near to the peak of the pulsed flow. Figure 5 represents the conditions during intervals of positive IMF $B_{y}$, which produces an enhancement of eastward flow on newly-opened field lines. This effect can be clearly seen from the flow streamline panels in the left column, with the effect being more pronounced for the fast model in the top two panels. For the case of northward IMF $B_{z}$ the fast flow model in the first row, we see that the asymmetry in the vortical flows associated with positive $B_{y}$ decreases the peak upward field-aligned current density to $\sim 0.28 \mu \mathrm{A} \mathrm{m}^{-2}$ near to the western end of the merging gap, and changes the shape of the region of upward current to a "spot" on the pre-noon side of the main auroral oval. The total current flowing over the rectangle $-6000 \mathrm{~km}$ to $0 \mathrm{~km}$ in $x$, and $\pm 350 \mathrm{~km}$ in $y$ is reduced to 0.47 MA. Conversely, in the downward current region at the post-noon end of the merging gap the peak field-aligned current density is increased to $\sim-1.04 \mu \mathrm{A} \mathrm{m}^{-2}$, and the total downward-directed current over the rectangle from $750 \mathrm{~km}$ to $3750 \mathrm{~km}$ in $x$, and $\pm 350 \mathrm{~km}$ in $y$ is increased also to $-0.40 \mathrm{MA}$. In the case of 


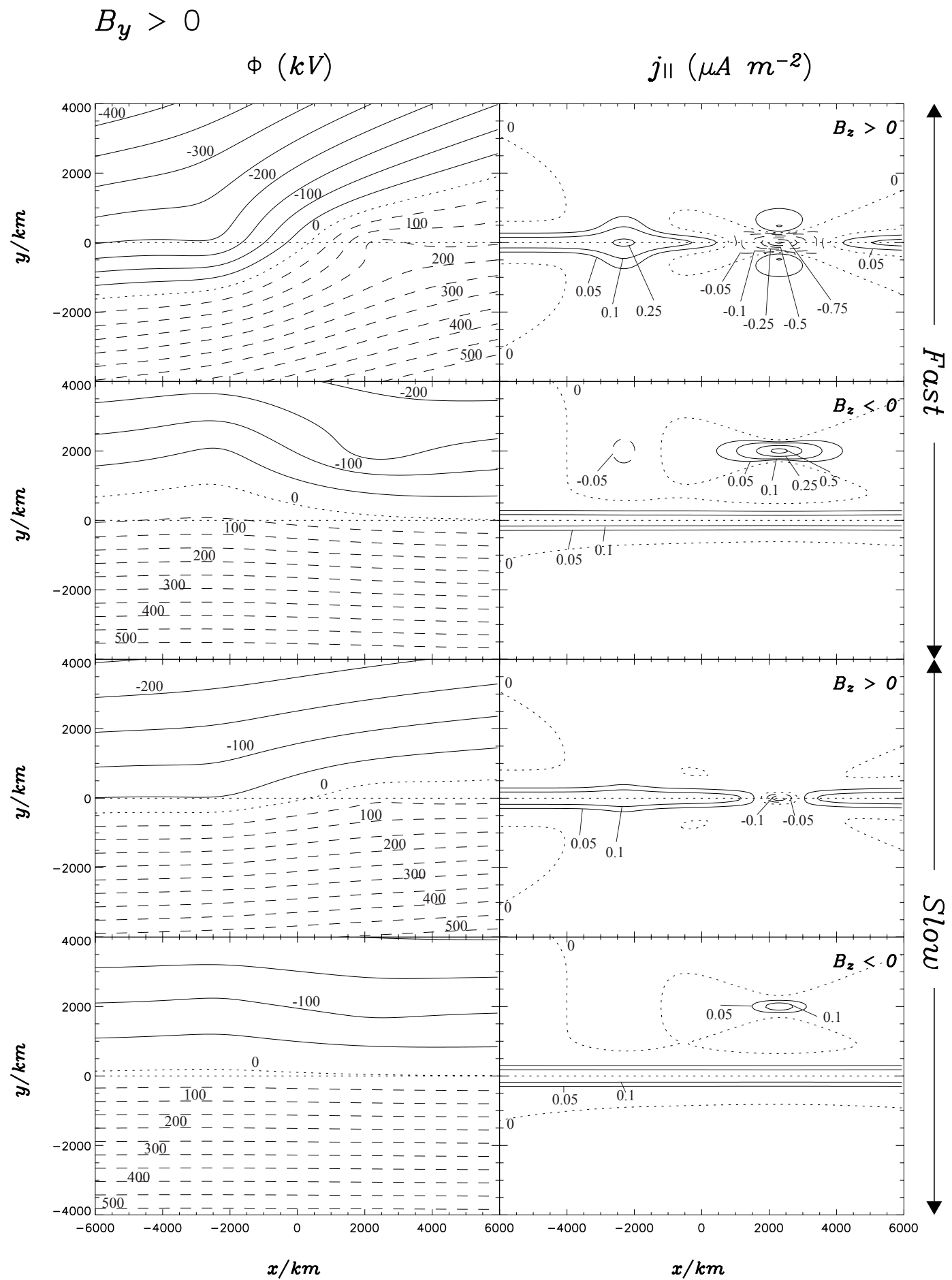

Fig. 5. In the same format as Fig. 3, contours of the electrostatic potential $\Phi_{i}$, and field-aligned current density $j_{\| i}$, are shown, but now for the case of $B_{y}>0$.

the slow flow model the same effect is seen, but to a lesser extent. The peak upward and downward field-aligned current densities are now 0.19 and $-0.14 \mu \mathrm{A} \mathrm{m}^{-2}$, respectively. The total current flowing is $0.44 \mathrm{MA}$ in the upward current rectangle and $-0.01 \mathrm{MA}$ in a modified downward current rectangle which ranges from 2000 to $2500 \mathrm{~km}$ in $x$ and $\pm 350 \mathrm{~km}$ in $y$. For the case of southward IMF $B_{z}$, the high-latitude reversed vortical flows and currents are similarly affected by the azimuthal flows due to IMF $B_{y}$. In the fast flow model the high-latitude region of upward field-aligned current density at the eastern end of the high-latitude merging gap has been intensified to a peak of $\sim 0.60 \mu \mathrm{A} \mathrm{m}^{-2}$, whilst the peak 


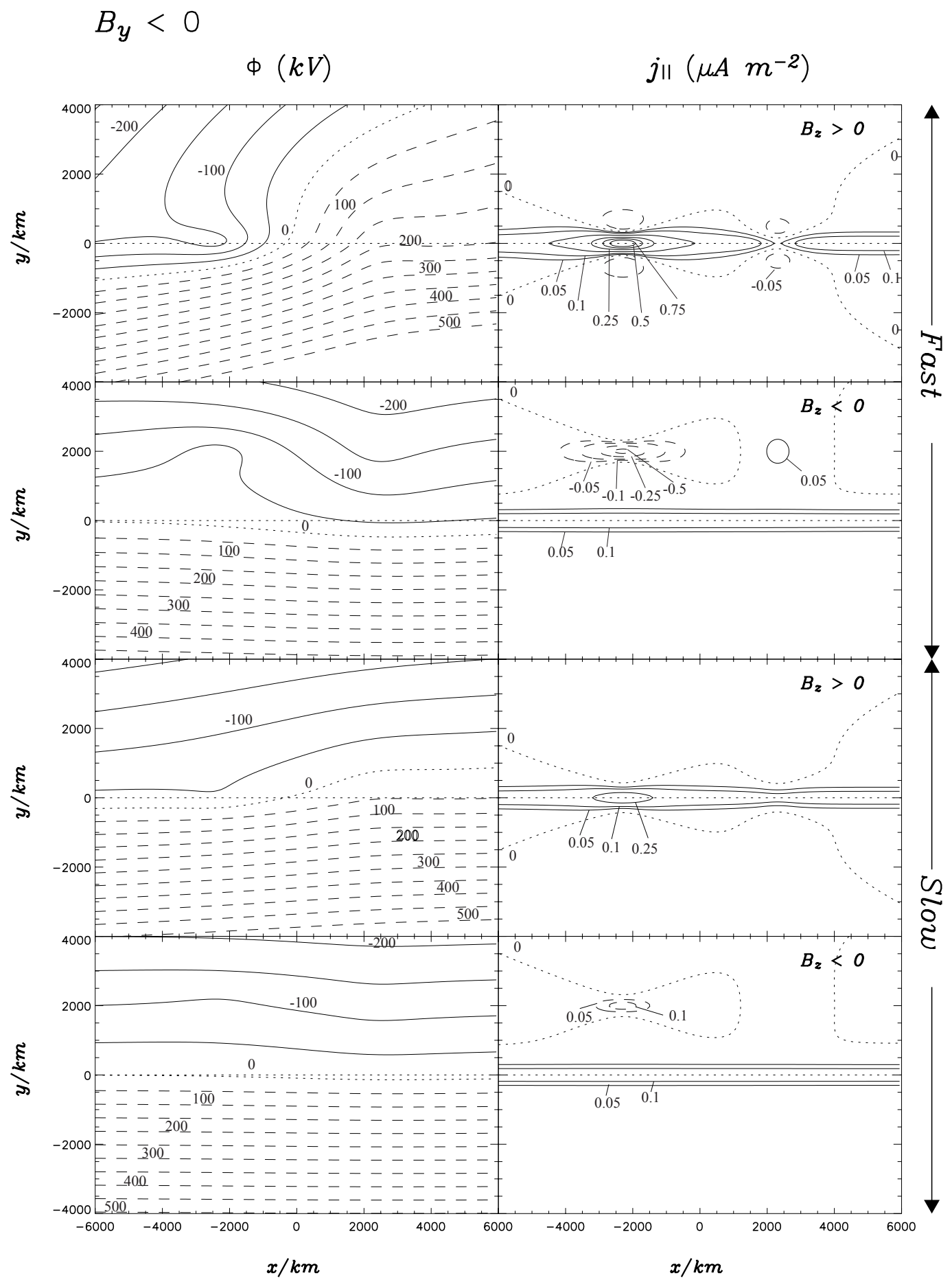

Fig. 6. In the same format as Fig. 3, plots of the electrostatic potential $\Phi_{i}$, and field-aligned current density $j_{\| i}$, are shown, but now for the case of $B_{y}<0$.

downward field-aligned current density has been decreased significantly to $-0.06 \mu \mathrm{A} \mathrm{m}^{-2}$. The total current flowing in the upward current region at the eastern end of the merging gap in the rectangle 1000 to $3500 \mathrm{~km}$ in $x$, and 1750 to $2250 \mathrm{~km}$ in $y$ is $0.25 \mathrm{MA}$. Conversely, in the downward current region at the western end of the merging gap between -3500 and $-1000 \mathrm{~km}$ in $x$, and 1750 and $2250 \mathrm{~km}$ in $y$, the total downward current is -0.06 MA. For the case of the slow flow model the effect is the same but less pronounced, the peak upward current density at the eastern end of the merging gap now being $0.15 \mu \mathrm{A} \mathrm{m}^{-2}$, whilst the peak downward current at the western end of the high-latitude merging gap is $-0.02 \mu \mathrm{A} \mathrm{m}^{-2}$. The total upward current in the slightly smaller rectangle between 1750 and $3000 \mathrm{~km}$ in $x$ 
and over 1750 to $2250 \mathrm{~km}$ in $y$ is $0.04 \mathrm{MA}$, whilst in the similarly modified rectangle in the downward current region it is $-0.01 \mathrm{MA}$.

In Fig. 6 we similarly present the flows and currents for the case of negative IMF $B_{y}$. In this case, the flow asymmetry introduces enhanced westward (or reduced eastward) flows on newly reconnected field lines, as can be seen from the flow streamline panels in the left column, and as previously, the effect is more pronounced for the fast model in the first two panels than for the slow flow model. For the case of northward IMF $B_{z}$ for the fast flow model in the first row, however, the flow perturbations actually produce a region of westward-directed flow in the inertial frame at the westward end of the merging gap. The peak upward fieldaligned current density in this model has increased from the $B_{y}=0$ case shown in Fig. 3 to $1.36 \mu \mathrm{A} \mathrm{m}^{-2}$ near the western end of the merging gap. The total upward current over the rectangle -6000 to $0 \mathrm{~km}$ in $x$ and $\pm 350 \mathrm{~km}$ in $y$ is $1.18 \mathrm{MA}$. Conversely, in the downward current region at the eastern end of the merging gap, the peak field-aligned current density is decreased to $-0.07 \mu \mathrm{A} \mathrm{m}^{-2}$. The total downward directed current over the rectangle 2000 to $2500 \mathrm{~km}$ in $x$ and $\pm 350 \mathrm{~km}$ in $y$ is $-0.01 \mathrm{MA}$. In the case of the slow flow model for IMF $B_{z}$ positive (third row) a similar but weaker effect is seen. The peak upward field-aligned current density at the western end of the merging gap is $0.46 \mu \mathrm{A} \mathrm{m}^{-2}$. Integrated over the rectangle -6000 to $0 \mathrm{~km}$ in $x$ and $\pm 350 \mathrm{~km}$ gives a total upward current of 0.6 MA. At the eastern end of the merging gap the downward-directed field-aligned current due to the clockwise vortex is unable to reverse the sense of the upward-directed field-aligned current on the openclosed field line boundary, as in the previous examples. However, the region of upward current on the boundary is somewhat narrower close to the end of the merging gap. For the case of southward IMF $B_{z}$, the high-latitude reversed vortical flows and currents are similarly affected by the azimuthal flows due to negative IMF $B_{y}$. In the fast flow model the high-latitude region of upward field-aligned current density at the eastern end of the high-latitude merging gap has been decreased to a "spot" of high-latitude field-aligned current peaking at a density of $\sim 0.07 \mu \mathrm{A} \mathrm{m}^{-2}$, whilst the peak downward field-aligned current density has been increased significantly to $-0.59 \mu \mathrm{A} \mathrm{m}^{-2}$. The total current flowing in the upward current region (within 1000 to $3500 \mathrm{~km}$ in $x$ and 1750 to $2250 \mathrm{~km}$ in $y$ as before) at the eastern end of the merging gap is 0.04 MA. In the downward directed current region, over the rectangle -3500 to $-1000 \mathrm{~km}$ in $x$ and 1750 to $2250 \mathrm{~km}$ in $y$, the total current is $-0.25 \mathrm{MA}$. For the case of the slow flow model the effect is similar with the peak upward current density at the eastern end of the merging gap now $0.02 \mu \mathrm{A} \mathrm{m}^{-2}$ (and hence is not shown) whilst the peak downward current at the western end of the highlatitude merging gap is $-0.15 \mu \mathrm{A} \mathrm{m}^{-2}$. The total current in the downward current region covering -3500 to $-1000 \mathrm{~km}$ in $x$ and 1750 to $2250 \mathrm{~km}$ in $y$ is $-0.06 \mathrm{MA}$.

\section{Field-aligned currents and auroral effects}

The conceptual flow models developed in the previous section suggest that pulses of dayside reconnection for varying orientations of the interplanetary magnetic field will produce transient pairs of upward- and downward-directed fieldaligned currents whose densities will lie in the range $\sim 0.01$ to $1 \mu \mathrm{A} \mathrm{m}^{-2}$. The geometry of these currents depends on the detailed form of the flows excited and the upstream IMF orientation, but generally cover an area a few hundred $\mathrm{km}$ in the $y$ direction and a few thousand $\mathrm{km}$ in the $x$ direction. Here we will consider the requirements for these currents to be carried by the ambient plasma populations in both open and closed field regimes, and for both sub-solar and high-latitude lobe reconnection. As indicated above, we choose to consider only the regions of upward-directed field-aligned currents (i.e. down-going electrons) for the following reasons. Down-going currents may in general be carried either by upgoing electrons or down-going precipitating ions, and generally we might suppose that the former dominates due to the high mobility of electrons, as is the case at the Earth. At Jupiter, however, evidence exists that the downward current in the cusp is carried at least partly by downward accelerated heavy ions, which produce both X-rays and bright UV auroras. At Saturn, there is no evidence of significant acceleration in the form of X-rays, and indeed the post-noon cusp is usually dark in the UV, where we might expect the downward currents to flow (Gérard et al., 2004). Thus we assume here that at Saturn the downward currents are carried principally by upward-flowing ionospheric electrons, and thus we concentrate on electron precipitation in upward current regions.

\subsection{Upward current regions during sub-solar reconnection}

Upward field-aligned currents during sub-solar reconnection (i.e. northwards IMF) are carried principally by downwardprecipitating electrons on outer magnetospheric field lines, specifically by hot trapped magnetospheric electrons on closed magnetic field lines, and by cool electrons originating from the sub-solar magnetosheath on newly-opened field lines. For an isotropic Maxwellian distribution of density $N$ and thermal energy $W_{t h}(=k T)$, the maximum field-aligned current density above the ionosphere that can be carried without field-aligned acceleration is

$$
j_{\| i_{0}}=e N\left(\frac{W_{t h}}{2 \pi m_{e}}\right)^{\frac{1}{2}},
$$

corresponding to a full downward-going loss cone, and an empty upward-going loss-cone. The precipitated energy flux in the same limit is

$$
E_{f_{0}}=2 N W_{t h}\left(\frac{W_{t h}}{2 \pi m_{e}}\right)^{\frac{1}{2}},
$$

this formula being the source of the energy flux estimates given in Sect. 2. For convenience, the source parameters 
Table 1. Source parameters employed for magnetosheath and outer magnetospheric plasmas on open and closed field lines and parameters derived from them for the case of sub-solar reconnection $\left(B_{z}>0\right)$. Also shown are the source parameters employed for the high-latitude plasma mantle and magnetosheath regions used in the case of lobe reconnection $\left(B_{z}<0\right)$. From the top, the rows of the table give information as follows:

\begin{tabular}{lllll}
\hline \multirow{2}{*}{ Source Parameter } & \multicolumn{2}{l}{ Sub-solar plasma population } & \multicolumn{2}{l}{ High-latitude population } \\
\cline { 2 - 5 } & $\begin{array}{l}\text { Low/high density } \\
\text { magnetosheath (open) }\end{array}$ & $\begin{array}{l}\text { Outer magnetosphere } \\
\text { (closed) }\end{array}$ & $\begin{array}{l}\text { Low/high density } \\
\text { magnetosheath (open) }\end{array}$ & $\begin{array}{l}\text { Low/high density } \\
\text { plasma mantle (open) }\end{array}$ \\
\hline$N\left(\mathrm{~cm}^{-3}\right)$ & $0.02 / 0.2$ & 0.01 & $0.01 / 0.1$ & $0.001 / 0.01$ \\
$W_{t h}(\mathrm{eV})$ & 50 & 600 & 25 & 25 \\
$j_{\| i 0}\left(\mathrm{nA} \mathrm{m}^{-2}\right)$ & $3.79 / 37.9$ & 8.47 & $1.34 / 13.4$ & $0.13 / 1.3$ \\
$E_{f 0}\left(\mu \mathrm{W} \mathrm{m}^{-2}\right)$ & $0.379 / 3.79$ & 17.0 & $0.067 / 0.67$ & $0.0067 / 0.067$ \\
\hline
\end{tabular}

1. $N$, the density of the source population in $\mathrm{cm}^{-3}$.

2. $W_{t h}$, the thermal energy of the source population in keV.

3. The limiting current at the ionosphere for the unaccelerated populations, determined from Eqs. (10) (Knight's (1973) Maxwellian formulation).

4. Limiting precipitating energy flux $E_{f_{0}}$, determined from Eq. (11).

employed here are collected together in Table 1, for typical conditions in the outer magnetosphere, and for the subsolar magnetosheath under intermediate (slow flow model) and high density (fast flow model) solar wind conditions. As discussed briefly in Sect. 2, the outer magnetospheric source, with typical densities of $0.01 \mathrm{~cm}^{-3}$ and thermal energies of $600 \mathrm{eV}$ (e.g. Richardson, 1995) produce limiting currents of $\sim 8.5 \mathrm{nA} \mathrm{m}^{-2}$ and energy fluxes of $\sim 17 \mu \mathrm{W} \mathrm{m}^{-2}$, respectively, as also indicated in Table 1 . The former is much less than the model currents described in Sect. 3, by factors of 10-100, and the energy fluxes are similarly very much lower than those required to explain the observed UV emissions discussed by Gerard et al. (2004), by significant factors, as discussed in Sect. 2. Therefore, significant fieldaligned acceleration of the magnetospheric electrons will be required to carry the model current densities described in Sect. 3, and also to produce any substantial UV emission. Consideration of the magnetosheath population on newlyopened field lines leads to a similar conclusion. For the slow flow model, typical densities of $\sim 0.005 \mathrm{~cm}^{-3}$ in the solar wind increase by a factor of $\sim 4$ to $\sim 0.02 \mathrm{~cm}^{-3}$ in the subsolar magnetosheath, with thermal energies $\sim 50 \mathrm{eV}$ (e.g. Sittler et al., 1983; Richardson and Sittler, 1990; Richardson, 1995). In this case the limiting current given by Eq. (10) is $\sim 3.8 \mathrm{nA} \mathrm{m}^{-2}$, with a limiting energy flux of $\sim 0.38 \mu \mathrm{W} \mathrm{m}^{-2}$ (see Table 1), so that similar conclusions apply. For the fast flow (high density solar wind) model, these latter values are increased by an order of magnitude, but nevertheless the limiting current is still significantly less than the peak upward field-aligned currents applicable to the model (see discussion in Sect. 3). We thus conclude that in both open and closed field regimes, downward acceleration of these electron populations is required.

In order to estimate the accelerating voltages required we use the kinetic theory of Knight (1973), who showed that when the field-aligned current at ionospheric heights is greater than $j_{\| i_{0}}$, the minimum accelerating voltage required is given by

$e \Phi_{\|}=W_{t h}\left[\left(\frac{j_{\| i}}{j_{\| i_{0}}}\right)-1\right]$.

This minimum voltage applies to the case where the voltage drop is located at a kronocentric radial distance significantly above the minimum possible distance, given by

$$
\left(\frac{r_{\text {min }}}{R_{J}}\right) \approx\left(\frac{j_{\| i}}{j_{\| i_{0}}}\right)^{1 / 3} .
$$

This minimum radial distance is determined by the requirement that a large enough flux of electrons must be present at the "top" of the voltage drop to form the current. In Eq. (13) it is assumed that the field strength falls as the cube of the distance along the polar field lines, corresponding to the dipole field of the planet. The corresponding energy flux of the accelerated precipitating electrons is then

$$
E_{f}=\frac{E_{f_{0}}}{2}\left[\left(\frac{j_{\| i}}{j_{\| i_{0}}}\right)^{2}+1\right],
$$

where $E_{f} 0$ is given by Eq. (11) (see Lundin and Sandahl, 1978). In Figs. $7 \mathrm{a}$ and $7 \mathrm{~b}$ we show the parallel voltage $\Phi_{\|}$and the precipitated energy flux $E_{f}$ versus the fieldaligned current density $j_{\| i}$, determined from these formulas for outer magnetospheric electrons (solid lines), low density magnetosheath electrons (dashed lines), and high density magnetosheath electrons (dotted lines). Results are shown for the relevant range of current densities, $0.01-0 \mu \mathrm{A} \mathrm{m}^{-2}$. Here we note that at a given current density $j_{\| i} \gg j_{\| i} 0$, the accelerating potential and precipitating energy flux is inversely proportional to the number density of the source population, such that the voltages and energy fluxes are higher for the 
more tenuous outer magnetospheric source than for either magnetosheath populations, and also larger for the low density magnetosheath than for the high density magnetosheath. Weaker effects are also present due to the difference in thermal energies, both $\Phi_{\|}$and $E_{f}$ scaling proportional to the square root of $W_{t h}$.

The consequences of the results presented in Fig. 7 may be understood by comparison with the upward-directed currents required by our slow and fast flow models discussed in the previous section, and shown in Figs. 3, 5, and 6 above. First we note that the boundary field-aligned current associated with the main auroral oval has a peak of $\sim 0.16 \mu \mathrm{A} \mathrm{m}^{-2}$ as discussed previously. Using the formulae above, this requires a field-aligned voltage of $\sim 20-30 \mathrm{kV}$, and produces a peak UV emission of $\sim 30 \mathrm{kR}$ on the closed side of the boundary. On the open side of the boundary in the fast model, a field-aligned voltage of $\sim 1 \mathrm{kV}$ is required to produce a peak $\mathrm{UV}$ emission of $\sim 0.3 \mathrm{kR}$, and in the slow model a peak voltage of $\sim 4 \mathrm{kV}$ is required to carry the current, and a peak $\mathrm{UV}$ emission of $\sim 3 \mathrm{kR}$ is produced. The reconnection related currents then modify these "main oval" currents as follows. For the case of sub-solar reconnection during northwards IMF and $B_{y}=0$, in the fast flow model the peak currents are $\sim 1.0 \mu \mathrm{A} \mathrm{m}^{-2}$. The enhanced field-aligned currents due to the pulsed reconnection are configured in a pre-noon arc along the open-closed field line boundary. Figure 7 then shows that on closed field lines a peak accelerating voltage of $\sim 88.0 \mathrm{kV}$ is required, producing a peak precipitating electron energy flux of $\sim 85.8 \mathrm{~mW} \mathrm{~m}^{-2}$, and a peak UV aurora of $\sim 859 \mathrm{kR}$ intensity. Simultaneously on open field lines, the peak accelerating voltage for the corresponding high density magnetosheath case is only $\sim 1.2 \mathrm{kV}$, producing a peak energy flux of $\sim 1.24 \mathrm{~mW} \mathrm{~m}^{-2}$, and hence a peak UV aurora of $\sim 12.4 \mathrm{kR}$. Similarly, the peak upward current density in the slow flow model for northwards IMF is $\sim 0.4 \mu \mathrm{A} \mathrm{m}^{-2}$, again in a pre-noon arc configuration as described for the fast flow model. This requires a peak voltage of $\sim 32.7 \mathrm{kV}$ on the closed field side of the boundary, resulting in a peak electron energy flux of $\sim 12.1 \mathrm{~mW} \mathrm{~m}^{-2}$, and $\sim 121 \mathrm{kR} \mathrm{UV}$ aurora. On the open field side of the boundary the peak accelerating voltage required for the corresponding low density magnetosheath case is $\sim 4.8 \mathrm{kV}$, with a maximum precipitated energy flux of $\sim 1.8 \mathrm{~mW} \mathrm{~m}^{-2}$, giving a maximum UV aurora of $\sim 18 \mathrm{kR}$.

Similarly, for the case of IMF $B_{y}>0$ (presented in Fig. 5), the upward-directed field-aligned currents are somewhat decreased in comparison to the case of $B_{y}=0$ as shown in Fig. 3 . For the fast model, we find that the peak field-aligned current is $\sim 0.3 \mu \mathrm{A} \mathrm{m}^{-2}$. The asymmetric flow directed eastwards in the Northern Hemisphere for positive IMF $B_{y}$ causes the geometry of the pulsed reconnection related emission to become more of a "spot" on the main auroral oval rather than the arc-like emission discussed above for $B_{y}=0$. On the closed side of the boundary, this requires a peak field-aligned voltage of $\sim 24.7 \mathrm{kV}$, and leads to a peak energy flux of $\sim 7.0 \mathrm{~mW} \mathrm{~m}^{-2}$ and peak UV emissions of $\sim 70.0 \mathrm{kR}$. In the open field regime, the corresponding high density solar wind
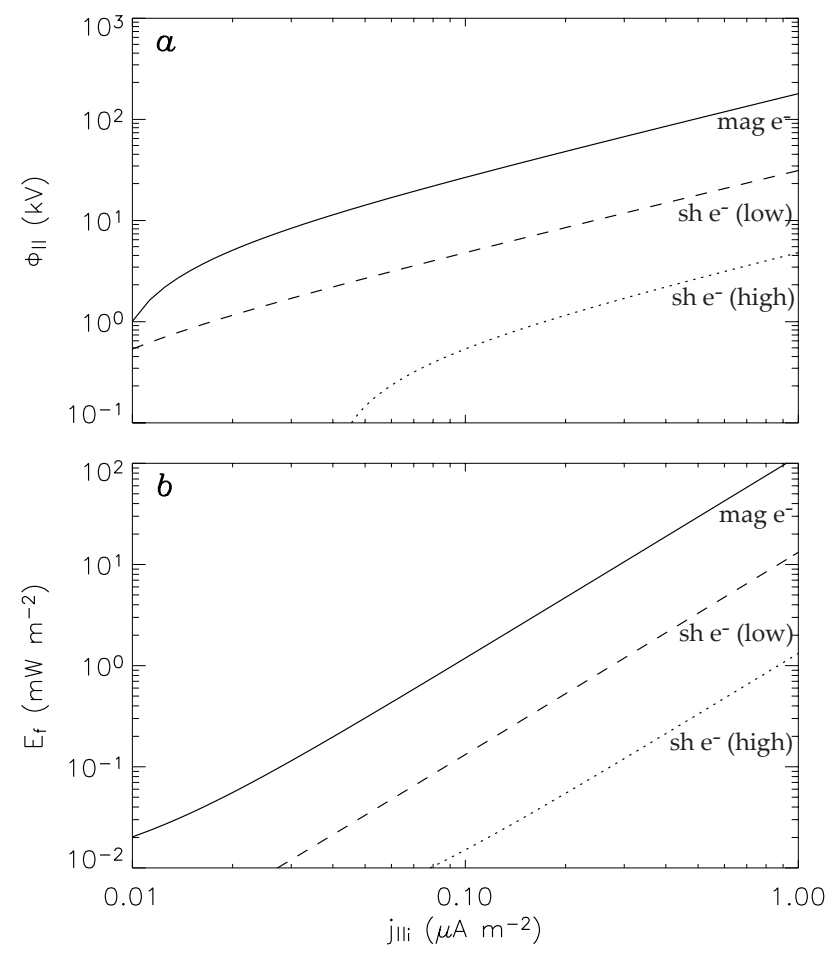

Fig. 7. Log-log plots of (a) the field-aligned voltage $\Phi_{\|}$in $\mathrm{kV}$, and (b) the accelerated energy flux $E_{f}$ in $\mathrm{mW} \mathrm{m}^{-2}$ as a function of field-aligned current density shown over the range of interest $0.01-1 \mu \mathrm{A} \mathrm{m}^{2}$ for precipitating electrons. These relations are relevant to the upward field-aligned current regions, obtained from Eqs. (12) and (14), for the electron populations present in the region of sub-solar reconnection (i.e. $B_{z}>0$ ). Here we show magnetospheric (solid lines), low-density magnetosheath (dashed lines) and high-density magnetosheath (dotted lines) electrons.

magnetosheath electrons require a peak voltage of $\sim 0.3 \mathrm{kV}$, giving peak accelerated energy flux of $\sim 0.1 \mathrm{~mW} \mathrm{~m}^{-2}$ and a maximum UV emission of $\sim 1.0 \mathrm{kR}$. In the slow model the emissions related to the $B_{y}>0$ case are not significantly elevated above the background currents, hence the peak currents are essentially those associated with the main auroral oval. On the closed side of the boundary, peak field-aligned current densities of $\sim 0.19 \mu \mathrm{A} \mathrm{m}^{-2}$ require peak accelerating voltages of $17.1 \mathrm{kV}$. This produces a peak energy flux of $\sim 3.4 \mathrm{~mW} \mathrm{~m}^{-2}$ and a peak UV emission of $\sim 34 \mathrm{kR}$. Equivalently, on the open side of the boundary, the corresponding low-density magnetosheath population requires a peak fieldaligned voltage of $\sim 2.5 \mathrm{kV}$. This implies peak energy flux values of $\sim 0.50 \mathrm{~mW} \mathrm{~m}^{-2}$ and hence $\sim 5 \mathrm{kR}$ of $\mathrm{UV}$ emission. These results are approximately those presented for the outer magnetosphere case in the Cowley et al. (2004) model.

For the case of IMF $B_{y}<0$ (presented in Fig. 6), the upward-directed field-aligned currents are enhanced in comparison to those shown in Fig. 3 for the case of $B_{y}=0$. For the case of the fast model, we find that the peak fieldaligned current is $\sim 1.4 \mu \mathrm{A} \mathrm{m}^{-2}$. The asymmetric flow directed westwards in the Northern Hemisphere for negative 
IMF $B_{y}$ causes the geometry of the pulsed reconnection related emission to become a more extended "arc" on the main auroral oval similar to the case shown for $B_{y}=0$. On the closed side of the boundary, this requires a peak field-aligned voltage of $\sim 124 \mathrm{kV}$, leading to a peak energy flux of $\sim 170 \mathrm{~mW} \mathrm{~m}^{-2}$ and hence peak UV emissions of $\sim 1.7$ MR. In the open field regime, the corresponding high density solar wind magnetosheath electrons require a peak voltage of $1.7 \mathrm{kV}$, giving peak accelerated energy flux of $\sim 2.5 \mathrm{~mW} \mathrm{~m}^{-2}$ and a maximum UV emission of $\sim 25 \mathrm{kR}$. In the slow flow model the peak field-aligned current densities are $\sim 0.5 \mu \mathrm{A} \mathrm{m}^{-2}$. On the closed side of the boundary this requires peak accelerating voltages of $\sim 41.5 \mathrm{kV}$. This produces a peak energy flux of $19.4 \mathrm{~mW} \mathrm{~m}^{-2}$ and a peak UV emission of $\sim 194 \mathrm{kR}$. On the open side of the boundary, the corresponding low-density magnetosheath population requires a peak field-aligned voltage of $\sim 6.0 \mathrm{kV}$. This implies peak energy flux values of $2.8 \mathrm{~mW} \mathrm{~m}^{-2}$ and hence up to $\sim 28 \mathrm{kR}$ of UV emission. For convenience the values discussed here are collected in Tables $2 \mathrm{a}$ and $\mathrm{b}$, in the column entitled "Sub-solar reconnection". The contents of these tables are described fully in the Appendix.

The results discussed above show that in both fast and slow model cases UV emission does indeed occur, though more significantly on closed field lines just equatorward of the open-closed field line boundary. The pre-noon arc emission which is formed along the main auroral oval itself, appears to be brightest for the IMF $B_{y}<0$ model, but with its geometry and intensity changing for various orientations of IMF $B_{y}$ as well as with fast and slow flow model input parameters. We have discussed above those values which are required by the peak field-aligned current densities in both our fast and slow flow conceptual models. Further information may be obtained by looking at integral values over the relevant areas associated with the field-aligned current contours discussed in Sect. 3. The areas of interest are shown in row (a) of Tables $2 a$ and $b$, and lead to the total currents which were also discussed in Sect. 3. In the fast model on closed outer magnetospheric field lines the principal region in the model extends from -6000 to $0 \mathrm{~km}$ in $x$, and -350 to $0 \mathrm{~km}$ in $y$ and provides an average UV emission over this area of $\sim 84 \mathrm{kR}$ for $B_{y}=0, \sim 15 \mathrm{kR}$ for $B_{y}>0$, and $123 \mathrm{kR}$ for $B_{y}<0$. The total precipitating power in each of these cases is $\sim 18,3$ and $26 \mathrm{GW}$ respectively. With $\sim 20 \%$ conversion efficiency as assumed here, the corresponding total UV output from these regions is thus $3.5,0.61$, and $5.2 \mathrm{GW}$ for the $B_{y}=0, B_{y}>0$, and $B_{y}<0$ models respectively. On the open side of the boundary, over the same area, the average UV emission is $1.2 \mathrm{kR}$ for $B_{y}=0,0.2 \mathrm{kR}$ for $B_{y}>0$, and $2 \mathrm{kR}$ for $B_{y}<0$. The total precipitating power in each of these cases is $\sim 0.25,0.04$ and $0.37 \mathrm{GW}$ respectively. The corresponding total UV output from these regions is thus $0.05,0.01$, and $0.08 \mathrm{GW}$ for the $B_{y}=0, B_{y}>0$, and $B_{y}<0$ models respectively. In the slow model on closed outer magnetospheric field lines the principal region in the model extends from -6000 to $0 \mathrm{~km}$ in $x$, and 0 to $350 \mathrm{~km}$ in $y$ and implies an average UV over this area of $\sim 22 \mathrm{kR}$ for $B_{y}=0, \sim 12 \mathrm{kR}$ for
$B_{y}>0$, and $26 \mathrm{kR}$ for $B_{y}<0$. The total precipitating power in each of these cases is $4.6,2.5$, and $5.4 \mathrm{GW}$ respectively. The corresponding total UV output from these regions is thus 0.9, 0.5 , and $1.1 \mathrm{GW}$ for the $B_{y}=0, B_{y}>0$, and $B_{y}<0$ models respectively. On the open side of the boundary, over the same area, the average UV emission is $\sim 3 \mathrm{kR}$ for $B_{y}=0, \sim 2 \mathrm{kR}$ for $B_{y}>0$, and $\sim 4 \mathrm{kR}$ for $B_{y}<0$. The total precipitating power in each of these cases is $0.7,0.4$, and $0.8 \mathrm{GW}$ respectively. The corresponding total UV output from these regions is thus $0.13, \sim 0.1$, and $\sim 0.15 \mathrm{GW}$ for the $B_{y}=0, B_{y}>0$, and $B_{y}<0$ models respectively. These results, and other relevant parameters, are also summarised in Tables $2 \mathrm{a}$ and $\mathrm{b}$.

\subsection{Upward current regions during high-latitude lobe re- connection}

Upward field-aligned currents produced by high-latitude reconnection in the lobes during intervals of southwards IMF $B_{z}$ are carried principally by downward-precipitating electrons originating from both the high-latitude magnetosheath and the plasma mantle in the lobe. As discussed briefly in Sect. 2 on the basis of the results e.g. of Spreiter et al. (1966), the high-latitude magnetosheath source is expected to have typical densities of about a half of those found in the sub-solar magnetosheath, thus typically in the range $0.01-0.1 \mathrm{~cm}^{-3}$ (e.g. Richardson, 1995). Similarly, thermal energies are likely to be around a half of those of the subsolar magnetosheath and will therefore typically have values of $25 \mathrm{eV}$ (e.g. Richardson, 1995). This population will thus produce limiting currents of $1.3-13.4 \mathrm{nA} \mathrm{m}^{-2}$ and energy fluxes of $0.07-0.7 \mu \mathrm{W} \mathrm{m}^{-2}$, respectively, as also indicated in Table 1. The former is much less than the model currents described in Sect. 3, by factors of 100-1000, and the energy fluxes are similarly very much lower than those required to explain the observed UV emissions. Therefore significant field-aligned acceleration of these electrons will be required to carry the model current densities described in Sect. 3, and also to produce substantial UV emission. Consideration of the plasma mantle population leads to similar conclusions. As discussed e.g. by Sckopke and Paschmann (1978) with regard to the Earth, we may reasonably expect the plasma mantle population to have densities which are a factor of ten lower than those in the high-latitude lobe magnetosheath, with temperatures that are similar. Therefore for simplicity, we consider the low and high density plasma mantle to have typical densities of $0.001-0.01 \mathrm{~cm}^{-3}$ and temperatures of $\sim 25 \mathrm{eV}$. This population will thus produce limiting currents of $0.1-1.3 \mathrm{nA} \mathrm{m}^{-2}$ and energy fluxes of 0.007$0.07 \mu \mathrm{W} \mathrm{m}^{-2}$, respectively, as also indicated in Table 1 . The former is essentially negligible compared to the model currents described in Sect. 3, and the energy fluxes are similarly very much lower than those required to explain the observed the UV emissions. We note that the low density magnetosheath and the high density plasma mantle population have the same densities and temperatures based on the discussion above. We thus once more conclude that substantial acceleration of the high-latitude electron population will be required 
in order to carry significant currents and to produce any UV emission.

In Figs. 8a and b, we show the parallel voltage $\Phi_{\|}$and the precipitated energy flux $E_{f}$ versus the field-aligned current density $j_{|| I}$, determined from Eq. (12) and (14), for the low density plasma mantle electrons (solid lines), high density plasma mantle electrons (dashed lines), low density highlatitude magnetosheath electrons (dashed lines), and high density high-latitude magnetosheath electrons (dotted lines). Results are shown, as in Fig. 5, for the relevant range of current densities, $0.01-1.0 \mu \mathrm{A} \mathrm{m}^{-2}$. The consequences of these results may be understood by comparison with the upwarddirected currents required by our slow and fast flow models for southwards IMF discussed in Sect. 3, and shown in Figs. 2, 4, and 5 above.

In the case of $B_{y}=0$, for the fast model the peak upward field-aligned current density at the eastern end of the highlatitude merging gap is $\sim 0.4 \mu \mathrm{A} \mathrm{m}^{-2}$. The geometry of these currents produce a "spot" of upward-directed fieldaligned current poleward of the continuous upward-directed field-aligned current thought to be responsible for the main auroral oval emissions. A peak field-aligned current density of this order leads to peak field-aligned voltages of $\sim 7.5 \mathrm{kV}$ for the high-density plasma mantle, resulting in a peak energy flux of $\sim 3.0 \mathrm{~mW} \mathrm{~m}^{-2}$, and a maximum UV aurora of $\sim 30 \mathrm{kR}$. For the high-density high-latitude magnetosheath source the equivalent field-aligned current density requires peak field aligned voltages of $\sim 0.7 \mathrm{kV}$, producing a peak energy flux of $\sim 0.3 \mathrm{~mW} \mathrm{~m}^{-2}$, and hence a peak UV emission of $\sim 3 \mathrm{kR}$. In the slow flow model, the field-aligned current density for the case of the high-latitude "spot" is weaker at $\sim 0.1 \mu \mathrm{A} \mathrm{m}^{-2}$. For the appropriate low-density plasma mantle population the peak field-aligned voltage required is $\sim 18.7 \mathrm{kV}$ corresponding to a maximum energy flux of $\sim 1.9 \mathrm{~mW} \mathrm{~m}^{-2}$, and a UV aurora of $\sim 20 \mathrm{kR}$. For the lowdensity high-latitude magnetosheath the equivalent fieldaligned current density requires peak field aligned voltages of $\sim 1.9 \mathrm{kV}$, producing a peak energy flux of $\sim 0.19 \mathrm{~mW} \mathrm{~m}^{-2}$, and hence a peak UV emission of $\sim 2 \mathrm{kR}$.

By comparison of Figs. 4 and 5 with Fig. 8, we can investigate the additional effects of asymmetries introduced by varying the direction of IMF $B_{y}$. For $B_{y}>0$, for the fast flow model the peak upward field-aligned current density at the eastern end of the high-latitude merging gap is intensified in comparison to the $B_{y}=0$ case discussed above to $\sim 0.6 \mu \mathrm{A} \mathrm{m}^{-2}$, and takes the form of an elongated "spot" poleward of the main auroral oval currents. A fieldaligned current density of this order leads to peak fieldaligned voltages of $\sim 11.0 \mathrm{kV}$ for the high-density plasma mantle source, resulting in a maximum electron energy flux of $\sim 6.6 \mathrm{~mW} \mathrm{~m}^{-2}$, and a peak UV aurora of $\sim 66 \mathrm{kR}$. For the high-density high-latitude magnetosheath source, the same field-aligned current density requires peak field-aligned voltages of $\sim 1.1 \mathrm{kV}$, peak energy fluxes of $\sim 0.7 \mathrm{~mW} \mathrm{~m}^{-2}$, leading to peak UV emission intensities of $\sim 7 \mathrm{kR}$. In the slow flow model, the peak field-aligned current density for the case of the high-latitude "spot" is $\sim 0.15 \mu \mathrm{A} \mathrm{m}^{-2}$. For the
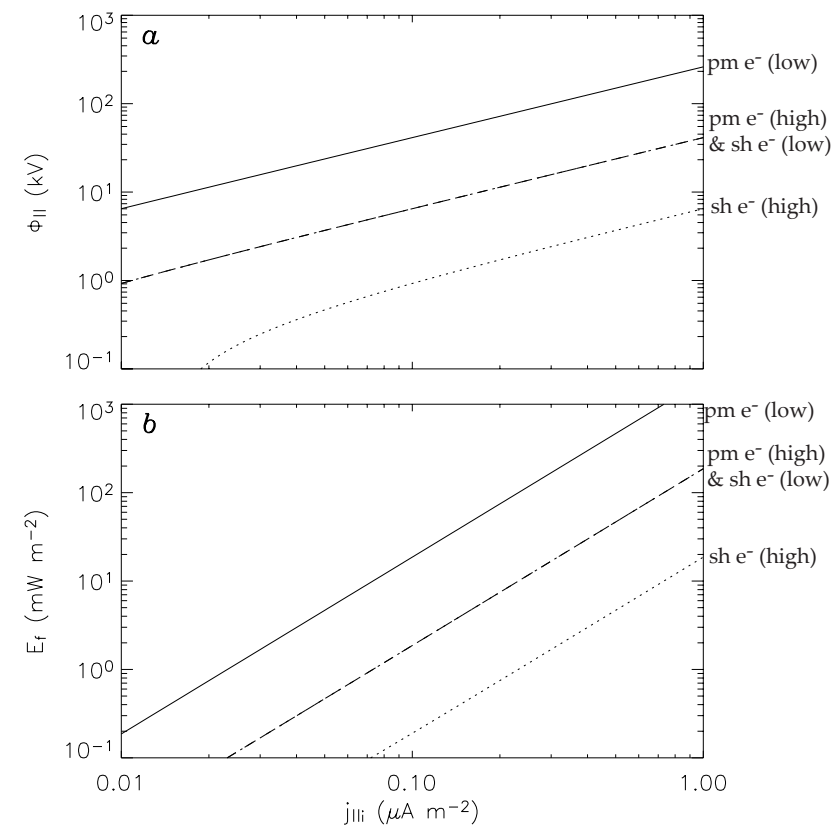

Fig. 8. Log-log plots of (a) the field-aligned voltage $\Phi_{\|}$in $\mathrm{kV}$, and (b) the accelerated energy flux $E_{f}$ in $\mathrm{mW} \mathrm{m}^{-2}$ as a function of field-aligned current density in the same format as Fig. 7, but now for the case of high-latitude lobe reconnection and the relevant electron populations. Here we show low- and high-density plasma mantle (solid line and dashed line respectively), and lowand high-density lobe magnetosheath (dashed line and dotted line respectively) electrons.

appropriate low-density plasma mantle population the peak field-aligned voltage required is $\sim 27.6 \mathrm{kV}$ corresponding to a peak energy flux of $\sim 4.1 \mathrm{~mW} \mathrm{~m}^{-2}$, and a maximum UV auroral emission of $\sim 41 \mathrm{kR}$. Finally, for the appropriate low-density high-latitude magnetosheath population the peak field-aligned voltage required is $\sim 2.7 \mathrm{kV}$, corresponding to a peak energy flux of $\sim 0.4 \mathrm{~mW} \mathrm{~m}^{-2}$, and a maximum $\mathrm{UV}$ aurora of $\sim 4 \mathrm{kR}$.

Finally in the IMF $B_{y}<0$ model (presented in Fig. 5) we find that the peak upward-directed field-aligned currents are now reduced significantly from those presented in Fig. 3 for the case of $B_{y}=0$. For $B_{y}<0$, for the fast flow model the peak upward field-aligned current density at the eastern end of the high-latitude merging gap is $\sim 0.06 \mu \mathrm{A} \mathrm{m}^{-2}$, once more in the form of a "spot" poleward of the main auroral oval currents. A field-aligned current density of this order leads to peak field-aligned voltages of $\sim 1.2 \mathrm{kV}$ for the high-density plasma mantle source, resulting in a maximum electron energy flux of $\sim 0.1 \mathrm{~mW} \mathrm{~m}^{-2}$, and a peak UV aurora of $\sim 1 \mathrm{kR}$. For the high-density high-latitude magnetosheath source, the same field-aligned current density requires peak field-aligned voltages of $\sim 0.1 \mathrm{kV}$, peak energy fluxes of $\sim 0.01 \mathrm{~mW} \mathrm{~m}^{-2}$, leading to peak UV emission intensities of $\sim 0.1 \mathrm{kR}$. In the slow flow model, the field-aligned current density for the case of the high-latitude "spot" is $\sim 0.01 \mu \mathrm{A} \mathrm{m}^{-2}$. For the appropriate low-density plasma mantle source population the 


\begin{tabular}{|c|c|c|c|c|c|}
\hline & \multirow{3}{*}{ Parameter } & \multicolumn{4}{|c|}{ Fast flow model } \\
\hline & & \multicolumn{2}{|c|}{ Sub-solar reconnection } & \multicolumn{2}{|c|}{ High-latitude reconnection } \\
\hline & & Outer magnetosphere & Magnetosheath & Plasma mantle & Magnetosheath \\
\hline$a$ & $\begin{array}{l}\text { Principal region } \\
\text { in model } \\
\text { Area of region }\end{array}$ & $\begin{array}{l}-6000 \leq x \leq 0 \mathrm{~km} \\
-350 \leq y \leq 0 \mathrm{~km} \\
2.1 \times 10^{12} \mathrm{~m}^{2}\end{array}$ & $\begin{array}{l}-6000 \leq x \leq 0 \mathrm{~km} \\
0 \leq y \leq 350 \mathrm{~km} \\
2.1 \times 10^{12} \mathrm{~m}^{2}\end{array}$ & $\begin{array}{l}1000 \leq x \leq 3500 \mathrm{~km} \\
1750 \leq y \leq 2000 \mathrm{~km} \\
6.3 \times 10^{11} \mathrm{~m}^{2}\end{array}$ & $\begin{array}{l}1000 \leq x \leq 3500 \mathrm{~km} \\
2000 \leq y \leq 2250 \mathrm{~km} \\
6.3 \times 10^{11} \mathrm{~m}^{2}\end{array}$ \\
\hline$b$ & $\left|j_{\| \mathrm{i}}\right|_{\max }\left(\mu \mathrm{A} \mathrm{m}^{-2}\right)$ & $1.0(0.28,1.36)$ & $1.0(0.28,1.36)$ & $0.40(0.60,0.06)$ & $0.40(0.60,0.06)$ \\
\hline$c$ & $\left\langle j_{\| \mathrm{i}}\right\rangle\left(\mu \mathrm{A} \mathrm{m} \mathrm{m}^{-2}\right)$ & $0.24(0.11,0.28)$ & $0.24(0.11,0.28)$ & $0.16(0.20,0.03)$ & $0.16(0.20,0.03)$ \\
\hline$d$ & $I_{\|}(\mathrm{MA})$ & $0.51(0.23,0.60)$ & $0.51(0.23,0.60)$ & $0.10(0.13,0.02)$ & $0.10(0.13,0.02)$ \\
\hline$e$ & $j_{\| \mathrm{i}_{0}}\left(\mathrm{nA} \mathrm{m}^{-2}\right)$ & 6.6 & 37.9 & 1.34 & 13.4 \\
\hline$f$ & $r_{\min } \max \left(\mathrm{R}_{\mathrm{S}}\right)$ & $5.3(3.5,5.9)$ & $2.9(1.9,3.3)$ & $6.7(7.6,3.6)$ & $3.1(3.5,1.7)$ \\
\hline$g$ & $\left\langle r_{\min }\right\rangle\left(\mathrm{R}_{\mathrm{S}}\right)$ & $3.3(2.6,3.5)$ & $1.9(1.4,2.0)$ & $5.0(5.3,2.8)$ & $2.3(2.5,1.3)$ \\
\hline$h$ & $\Phi_{\|_{\max }}(\mathrm{kV})$ & $88.0(24.7,123.9)$ & $1.2(0.3,1.7)$ & $7.5(11.0,1.2)$ & $0.73(1.1,0.1)$ \\
\hline$i$ & $\left\langle\Phi_{\|}\right\rangle(\mathrm{kV})$ & $33.9(12.5,42.8)$ & $0.4(0.1,0.6)$ & $3.9(5.3,0.8)$ & $0.4(0.5,0.06)$ \\
\hline$j$ & $E_{\mathrm{f}_{\max }}\left(\mathrm{mW} \mathrm{m}^{-2}\right)$ & $85.8(7.0,169.7)$ & $1.24(0.10,2.45)$ & $3.03(6.6,0.10)$ & $0.30(0.7,0.01)$ \\
\hline$k$ & $\left\langle E_{\mathrm{f}}\right\rangle\left(\mathrm{mW} \mathrm{m}^{-2}\right)$ & $8.4(1.5,12.3)$ & $0.12(0.02,0.17)$ & $0.65(1.2,0.02)$ & $0.07(0.11,0.002)$ \\
\hline$l$ & $P_{\mathrm{E}_{\mathrm{f}}}(\mathrm{GW})$ & $17.6(3.0,25.9)$ & $0.25(0.04,0.37)$ & $0.41(0.67,0.02)$ & $0.04(0.07,0.002)$ \\
\hline$m$ & $I_{U V_{\max }}(\mathrm{kR})$ & $858.9(70.3,1697.0)$ & $12.4(1.0,24.5)$ & $30.3(65.7,0.76)$ & $3.0(6.6,0.08)$ \\
\hline$n$ & $\left\langle I_{\mathrm{UV}}\right\rangle(\mathrm{kR})$ & $83.8(14.6,123.2)$ & $1.2(0.21,1.8)$ & $6.5(10.7,0.24)$ & $0.70(1.1,0.02)$ \\
\hline$o$ & $P_{\mathrm{UV}}(\mathrm{GW})$ & $3.5(0.61,5.2)$ & $0.05(0.01,0.08)$ & $0.10(0.13,0.003)$ & $0.01(0.013,0.001)$ \\
\hline
\end{tabular}

Table 2. (a) Table summarizing the results obtained for our conceptual model of the pulsed kronian cusp, for regions of upward current on open and closed field lines for sub-solar reconnection, and for regions of open field lines in the lobe magnetosheath and the plasma mantle in the case of the high-latitude reconnection model. Results are shown here for our "fast flow" model, taken to be most appropriate to high density, high field solar wind conditions. For details of how the entries in this table were derived, see the Appendix. 


\begin{tabular}{|c|c|c|c|c|c|}
\hline & \multirow{3}{*}{ Parameter } & \multicolumn{4}{|c|}{ Slow flow model } \\
\hline & & \multicolumn{2}{|c|}{ Sub-solar reconnection } & \multicolumn{2}{|c|}{ High-latitude reconnection } \\
\hline & & Outer magnetosphere & Magnetosheath & Plasma mantle & Magnetosheath \\
\hline$a$ & $\begin{array}{l}\text { Principal region } \\
\text { in model } \\
\text { Area of region }\end{array}$ & $\begin{array}{l}-6000 \leq x \leq 0 \mathrm{~km} \\
-350 \leq y \leq 0 \mathrm{~km} \\
2.1 \times 10^{12} \mathrm{~m}^{2}\end{array}$ & $\begin{array}{l}-6000 \leq x \leq 0 \mathrm{~km} \\
0 \leq y \leq 350 \mathrm{~km} \\
2.1 \times 10^{12} \mathrm{~m}^{2}\end{array}$ & $\begin{array}{l}1750 \leq x \leq 3000 \mathrm{~km} \\
1750 \leq y \leq 2000 \mathrm{~km} \\
3.1 \times 10^{11} \mathrm{~m}^{2}\end{array}$ & $\begin{array}{l}1750 \leq x \leq 3000 \mathrm{~km} \\
2000 \leq y \leq 2250 \mathrm{~km} \\
3.1 \times 10^{11} \mathrm{~m}^{2}\end{array}$ \\
\hline$b$ & $\left|j_{|| \mathrm{i}}\right|_{\max }\left(\mu \mathrm{A} \mathrm{m}^{-2}\right)$ & $0.36(0.19,0.46)$ & $0.36(0.19,0.46)$ & $0.10(0.15,0.01)$ & $0.10(0.15,0.01)$ \\
\hline$c$ & $\left\langle j_{\| \mathrm{i}}\right\rangle\left(\mu \mathrm{A} \mathrm{m} \mathrm{m}^{-2}\right)$ & $0.14(0.11,0.15)$ & $0.14(0.11,0.15)$ & $0.06(0.07,0.01)$ & $0.06(0.07,0.01)$ \\
\hline$d$ & $I_{\|}(\mathrm{MA})$ & $0.29(0.22,0.31)$ & $0.29(0.22,0.31)$ & $0.02(0.02,0.003)$ & $0.02(0.02,0.003)$ \\
\hline$e$ & $j_{\| \mathrm{i}_{0}}\left(\mathrm{nA} \mathrm{m}^{-2}\right)$ & 6.6 & 3.79 & 0.13 & 1.34 \\
\hline$f$ & $r_{\min }{ }_{\max }\left(\mathrm{R}_{\mathrm{S}}\right)$ & $3.8(3.1,4.1)$ & $4.6(3.7,5.0)$ & $9.0(10.3,5.0)$ & $4.2(4.8,2.3)$ \\
\hline$g$ & $\left\langle r_{\min }\right\rangle\left(\mathrm{R}_{\mathrm{S}}\right)$ & $2.8(2.5,2.8)$ & $3.3(3.0,3.4)$ & $7.4(8.0,4.5)$ & $3.5(3.7,2.1)$ \\
\hline$h$ & $\Phi_{\|_{\max }}(\mathrm{kV})$ & $32.7(17.1,41.5)$ & $4.8(2.5,6.0)$ & $18.7(27.6,3.0)$ & $1.9(2.7,0.27)$ \\
\hline$i$ & $\left\langle\Phi_{\|}\right\rangle(\mathrm{kV})$ & $15.1(10.7,16.8)$ & $2.2(1.6,2.5)$ & $11.9(16.2,2.3)$ & $1.2(1.6,0.2)$ \\
\hline$j$ & $E_{\mathrm{f}_{\max }}\left(\mathrm{mW} \mathrm{m}^{-2}\right)$ & $12.1(3.41,19.4)$ & $1.8(0.50,2.79)$ & $1.90(4.1,0.05)$ & $0.19(0.41,0.005)$ \\
\hline$k$ & $\left\langle E_{\mathrm{f}}\right\rangle\left(\mathrm{mW} \mathrm{m}{ }^{-2}\right)$ & $2.2(1.20,2.56)$ & $0.31(0.17,0.37)$ & $0.70(1.12,0.03)$ & $0.07(0.11,0.003)$ \\
\hline$l$ & $P_{\mathrm{E}_{\mathrm{f}}}(\mathrm{GW})$ & $4.6(2.5,5.4)$ & $0.7(0.4,0.8)$ & $0.21(0.35,0.01)$ & $0.02(0.03,0.001)$ \\
\hline$m$ & $I_{\mathrm{UV}_{\max }}(\mathrm{kR})$ & $121.2(34.1,193.9)$ & $17.5(4.9,27.9)$ & $18.9(41.0,0.47)$ & $1.9(4.1,0.05)$ \\
\hline$n$ & $\left\langle I_{\mathrm{UV}}\right\rangle(\mathrm{kR})$ & $21.7(12.0,25.6)$ & $3.1(1.7,3.7)$ & $6.6(11.2,0.28)$ & $0.7(1.1,0.03)$ \\
\hline$o$ & $P_{\mathrm{UV}}(\mathrm{GW})$ & $0.90(0.50,1.10)$ & $0.13(0.07,0.15)$ & $0.04(0.17,0.002)$ & $0.005(0.006,0.0002)$ \\
\hline
\end{tabular}

Table 2. (b) Table summarizing the results obtained for our conceptual model of the pulsed kronian cusp, in the same format as Table $2 \mathrm{a}$, but for the case of our "slow flow" model, taken to be most appropriate to intermediate density, intermediate field strength solar wind conditions. For details of how the entries in this table were derived, see the Appendix. 
peak field-aligned voltage required is $\sim 3.0 \mathrm{kV}$ corresponding to a peak energy flux of $\sim 0.05 \mathrm{~mW} \mathrm{~m}^{-2}$, and a maximum UV aurora of $\sim 0.5 \mathrm{kR}$. For the low-density high-latitude magnetosheath population the peak field-aligned voltage required is $\sim 0.3 \mathrm{kV}$ corresponding to a peak energy flux of $\sim 0.005 \mathrm{~mW} \mathrm{~m}^{-2}$, and a maximum UV aurora of $\sim 0.05 \mathrm{kR}$. Again, the values discussed here are collected in Tables 2a and $b$, in the column labelled "High-latitude reconnection". Information on how these parameters were derived is given in the Appendix.

The results discussed above show that in both fast and slow flow model cases, therefore, UV emission indeed occurs, in the form of a "spot" poleward of the main auroral oval itself. The UV intensity is enhanced for the case of IMF $B_{y}>0$ compared with $B_{y}=0$, and reduced for $B_{y}<0$. The UV emission is also greatest for the case of the plasma mantle electron population, rather than for the high-latitude magnetosheath source. Again, we have only so far discussed those values which are required by the peak field-aligned current densities in both our fast and slow flow conceptual models. A more overall impression may be obtained by looking at the relevant areas associated with the field-aligned current contours discussed in Sect. 3. The areas of interest are shown in row (a) of Tables 2a and b, and lead to the total currents which were also discussed in Sect. 3. In the fast model with the plasma mantle source the principal region in the model extends from 1000 to $3000 \mathrm{~km}$ in $x$, and 1750 to $2000 \mathrm{~km}$ in $y$ and gives an average UV intensity over this area of $\sim 6.5 \mathrm{kR}$ for $B_{y}=0, \sim 10.7 \mathrm{kR}$ for $B_{y}>0$, and $0.25 \mathrm{kR}$ for $B_{y}<0$. The total precipitating power in each of these cases is $0.4,0.7$, and 0.02 GW respectively. With $\sim 20 \%$ conversion efficiency as assumed here, the corresponding total UV output from these regions is thus $0.1,0.13$, and $0.003 \mathrm{GW}$ for the $B_{y}=0, B_{y}>0$, and $B_{y}<0$ models respectively. For the high-latitude magnetosheath source the principal region extends from 1000 to $3500 \mathrm{~km}$ in $x$, and 2000 to $2250 \mathrm{~km}$ in $y$ and gives an average UV intensity over this area of $\sim 0.7 \mathrm{kR}$ for $B_{y}=0, \sim 1.1 \mathrm{kR}$ for $B_{y}>0$, and $0.02 \mathrm{kR}$ for $B_{y}<0$, the latter being beneath the limit of detection by HST. The total precipitating power in each of these cases is $0.04,0.07$, and $0.002 \mathrm{GW}$ respectively. The corresponding total UV output from these regions is thus $0.01,0.013$, and $0.001 \mathrm{GW}$ for the $B_{y}=0, B_{y}>0$, and $B_{y}<0$ models respectively. In the slow flow model for the plasma mantle source the principal region in the model extends from 1750 to $3000 \mathrm{~km}$ in $x$, and 1750 to $2000 \mathrm{~km}$ in $y$ and gives an average UV over this area of $\sim 6.6 \mathrm{kR}$ for $B_{y}=0, \sim 11.2 \mathrm{kR}$ for $B_{y}>0$, and $0.3 \mathrm{kR}$ for $B_{y}<0$. The total precipitating power in each of these cases is $0.2,0.4$, and $0.01 \mathrm{GW}$ respectively. The corresponding total UV output from these regions is thus 0.04, 0.07, and $0.002 \mathrm{GW}$ for the $B_{y}=0, B_{y}>0$, and $B_{y}<0$ models respectively. Finally, for the slow flow model and the high-latitude magnetosheath source the principal region in the model extends from 1750 to $3000 \mathrm{~km}$ in $x$, and 2000 to $2250 \mathrm{~km}$ in $y$ and gives an average UV output over this area of $\sim 0.7 \mathrm{kR}$ for $B_{y}=0, \sim 1.1 \mathrm{kR}$ for $B_{y}>0$, and $0.03 \mathrm{kR}$ for $B_{y}<0$, the latter again being beneath the limit of detection by HST. The total precipitating power in each of these cases is $0.02,0.03$, and $0.001 \mathrm{GW}$ respectively. The corresponding total UV output from these regions is thus $0.005,0.006$, and $0.0002 \mathrm{GW}$ for the $B_{y}=0, B_{y}>0$, and $B_{y}<0$ models respectively. These results are also summarised in Tables $2 \mathrm{a}$ and b.

\section{Summary and discussion}

Recently, Gérard et al. (2004) have discussed dayside UV emissions in Saturn's polar ionosphere which they suggest may be the first observational evidence of the kronian "cusp". They describe the emission as having two distinct states. The first is a bright arc-like feature located in the pre-noon sector, and the second is a more diffuse "spot" of aurora which lies poleward of the general location of the main auroral oval. The morning arc emission varies in intensity up to $\sim 75 \mathrm{kR}$, accompanied often by a decreased level of emission on the afternoon oval. The higher-latitude "spot" has a lower intensity between $\sim 10-20 \mathrm{kR}$. The authors conjecture that this variability in location may be accounted for by the change of the IMF orientation from northwards to southwards, and the subsequent relocation of the merging gap to higher latitudes, as is seen at Earth (Maezawa, 1976; Burke et al., 1979; Heelis et al, 1986; Farrugia et al, 1995; Øieroset et al., 1997; Sandholt et al., 1998; Milan et al., 2000; Phan et al., 2004).

Here we have taken up this suggestion, first noting, however, that direct precipitation of electrons in the cusp regions are not capable of producing significant UV aurora. We therefore suggest that field-aligned acceleration of the plasma electrons following reconnection is required to account for the observed UV emissions. We have therefore investigated the possibility that the UV emissions observed by Gérard et al. (2004) are associated with reconnection occurring at the dayside magnetopause, possibly pulsed, akin to flux transfer events seen at the Earth. We have made a simple estimate of the reconnection interpulse period of $\sim 20-30$ min at Saturn, and suggest that there may be various signatures displaying this characteristic period, as we have suggested for the case of Jupiter (Bunce et al., 2004). As recently discussed for the case of the jovian cusp by Bunce et al. (2004), pulsed reconnection at the low-latitude dayside magnetopause for the case of northwards IMF will give rise to pulsed twin-vortical flows in the magnetosphere and ionosphere in the vicinity of the open-closed field-line boundary, and hence to bi-polar field-aligned currents centred in the vortical flows which close at one end in ionospheric Pedersen currents and at the other in the Chapman-Ferraro currents on the magnetopause. In the case of high-latitude lobe reconnection for southward IMF, we also expect to have pulsed twin-vortical flows and corresponding bi-polar field-aligned currents. However, the vortical flows in this case are displaced poleward of the open-closed field line boundary, and are reversed in sense, such that the field-aligned currents are also reversed compared with the former case. It should be noted here that these perturbation currents associated with pulsed reconnection at the magnetopause will straddle (for 
the case of northward IMF) or sit slightly poleward of (for the case of southward IMF) the open-closed field line boundary depicted in Fig. 1c. As discussed in the Introduction, and as presented by Cowley et al. (2004), the steady-state flows associated with the Dungey-cycle interaction at Saturn produce a ring of continuously upward field-aligned current along the open-closed field line boundary which they suggest can account for the main oval auroral emissions at Saturn, producing a few tens of kR emission. For both cases of northward and southward IMF we have also for the first time included the effects associated with the IMF $B_{y}$ effect. In the Northern Hemisphere, positive $B_{y}$ will lead to an associated azimuthal flow in the ionosphere which is directed eastwards, while conversely negative $B_{y}$ will lead to an azimuthal flow directed westwards. This effect is independent of the orientation of IMF $B_{z}$, and acts to enhance the eastward flows on open field lines in the case of IMF $B_{y}>0$, and reduces the eastward flow for the case of IMF $B_{y}<0$ (see Fig. 4). Of course, in the Southern Hemisphere the flows associated with IMF $B_{y}$ will be reversed, leading to an enhancement of the eastward flows on open field lines during intervals IMF $B_{y}<0$, and a reduction of the eastward flows on open field lines during intervals of IMF $B_{y}>0$.

In addition to the variability in flows and currents expected to be present due to changes in the orientation of the IMF, modulation is also introduced by the structured nature of the solar wind and IMF at Saturn's orbit (see Jackman et al., 2004). As at Jupiter, the highly developed nature of CIRs causes the solar wind and IMF to be separated into distinct regions of rarefaction and compression. We have recognised this variability in our conceptual model, and include a slow and fast flow model for the case of each IMF orientation. The slow flow model is chosen specifically to represent the intermediate levels of field strength and plasma density often observed during part of the rarefaction region (as opposed to the weakest field strengths of the rarefaction regions), whilst the latter represents the high-density and high-field strengths present during compression regions (see Jackman et al., 2004). In fact we can directly estimate the dayside reconnection rate from the IMF data, using the empirically based formulation discussed by Jackman et al. (2004). Using the data presented therein, we estimate voltages associated with the intermediate IMF density and strength to typically be $\sim 100 \mathrm{kV}$ and therefore use this to represent the voltage across the merging gap for our slow flow model. During high density, high field strength compression regions, the associated voltages peak at $\sim 400 \mathrm{kV}$, and we use this to represent the voltage across the merging gap for our fast flow model. The fast flow model thus represents the more extreme case. In each case the centres of the vortices are separated by $\sim 5000 \mathrm{~km}$ (i.e. $\sim 1 \mathrm{~h}$ of local time), so that the vortical flows speeds are $\sim 0.7 \mathrm{~km} \mathrm{~s}^{-1}$ in the fast model, and $0.175 \mathrm{~km} \mathrm{~s}^{-1}$ in the slow model. With a reasonable estimate of the ionospheric Pedersen conductivity of $\sim 1.0$ mho, the peak fieldaligned current densities and total currents in the fast flow model are $\sim 0.83 \mu \mathrm{A} \mathrm{m}^{-2}$ and $0.60 \mathrm{MA}$, and $\sim 0.21 \mu \mathrm{A} \mathrm{m}^{-2}$ and $0.15 \mathrm{MA}$ in the slow flow model. These currents mod- ulate the boundary current in the northward IMF case, taken to be a combination of near-stagnant anti-sunward flow and sub-corotational flow at the level of $\sim 30 \%$ of rigid corotation on open field lines, and $\sim 80 \%$ corotation on closed field lines, as recently discussed by Cowley et al. (2004). For the case of southward IMF, the voltages across the merging gap are taken to be approximately a half of that expected for northward IMF (Milan, 2004; Milan et al., 2004). The peak field-aligned currents and total currents in the fast flow model are $\sim 0.41 \mu \mathrm{A} \mathrm{m}^{-2}$ and $0.21 \mathrm{MA}$, and $0.11 \mu \mathrm{A} \mathrm{m}^{-2}$ and $0.05 \mathrm{MA}$ for the slow flow model, respectively. The vortical currents associated with high-latitude reconnection during intervals of southward IMF do not modulate the boundary current associated with the main auroral oval, as they are located significantly poleward.

We have then considered the conditions under which the plasma populations appropriate to either sub-solar reconnection or high-latitude lobe reconnection can carry the currents indicated. We have estimated the field-aligned voltages required, the resulting precipitating particle energy fluxes, and the consequent auroral output. We have assumed the fieldaligned currents described here are carried wholly by electrons, i.e. that downward-directed currents are carried by upgoing ionospheric electrons. Here, we are, therefore, interested only in regions of upward field-aligned current and downward accelerated electrons. Field-aligned voltages are required under all circumstances to carry the estimated currents, although the details vary between our fast and slow models, the cases for various IMF orientations, and the differing source populations involved. During sub-solar reconnection we find that on the closed side of the boundary voltages are $\sim 10-45 \mathrm{kV}$ above the region of upward-directed current, carried by outer magnetospheric electrons. On the open side of the boundary, the field-aligned voltages are estimated to be between $\sim 0.1-2.5 \mathrm{kV}$ above the region of upward current, carried by the cooler more dense magnetosheath electrons. In our model for high-latitude lobe reconnection, the voltages required to accelerate the plasma mantle electrons range from $\sim 1-17 \mathrm{kV}$ above the region of upward field-aligned current associated with the reversed high-latitude vortical flows. Finally, for the case of the highlatitude magnetosheath source, we estimate voltages between $\sim 0.1-1.6 \mathrm{kV}$ above the region of upward current. The precipitating energy flux and consequent UV output is then essentially given by the product of these voltages and the field-aligned current densities which are flowing, as outlined above.

In the case of sub-solar reconnection and IMF $B_{y}=0$, in the upward current region on the closed side of the boundary over an area of $2.1 \times 10^{12} \mathrm{~m}^{2}$, centred near the western end of the merging gap the averaged UV emission is $\sim 84 \mathrm{kR}$ in the fast model and $\sim 22 \mathrm{kR}$ in the slow model. The total UV output power in each case is $3.5 \mathrm{GW}$ and $1.0 \mathrm{GW}$ respectively. On the open side of the boundary the fast model produces $\sim 1.2 \mathrm{kR}$ averaged UV emission over the same area, and the slow model gives $\sim 3 \mathrm{kR}$. Again, the total UV power output is $0.05 \mathrm{GW}$ and $0.13 \mathrm{GW}$ respectively. The geometry 
of this emission takes the form of an arc in the pre-noon cusp location, in good agreement with the observations discussed by Gérard et al. (2004). In the post-noon region there is a region of downward current, which if carried by upgoing electrons will account for the "gap" in the oval discussed by Gérard et al. (2004). For high-latitude reconnection and IMF $B_{y}=0$, the merging gap is moved to higher latitudes (by $2000 \mathrm{~km}$ ), and produces UV emissions in the upward current region at the eastern end of the merging gap over an area of $6.3 \times 10^{11} \mathrm{~m}^{2}$ in the fast model and $3.1 \times 10^{11} \mathrm{~m}^{2}$ in the slow model. The geometry of this emission then takes the form of a high-latitude "spot", poleward of the main auroral oval currents. Both precipitating plasma mantle electrons and lobe magnetosheath electrons will carry the upward field-aligned currents into the ionosphere. In the fast model, the former produce an average of $\sim 7 \mathrm{kR}$ whilst in the slow model the average over the slightly reduced area is also $\sim 7 \mathrm{kR}$. The total UV output power in each case is then 0.1 and $0.04 \mathrm{GW}$ respectively. The high-latitude magnetosheath electrons are capable of producing $\sim 0.7 \mathrm{kR}$ in the fast model and approximately the same with the slow model and total UV output powers of just 0.01 and $0.005 \mathrm{GW}$ respectively. For the case of IMF $B_{y}>0$, the numbers quoted above are somewhat reduced for the case of sub-solar reconnection, where the upward field-aligned currents located at the western end of the merging gap (i.e. pre-noon) have been reduced by the additional azimuthal flows. Conversely, for the case of highlatitude reconnection, the upward currents at the eastern end of the merging gap are increased for IMF $B_{y}>0$, such that we might expect to see the brightest high-latitude "spot" emissions during intervals of $B_{z}<0$ and positive $B_{y}$. The opposite is true for the case of $B_{y}<0$, such that we would expect to see the brightest pre-noon arc for the combination of subsolar reconnection (i.e. $B_{z}>0$ ) and negative $B_{y}$. As mentioned above, the $B_{y}$ related ionospheric flows will be reversed in the Southern Hemisphere, such that it follows that the brightest cusp auroras may be associated with $B_{z}>0$ and $B_{y}>0$, and $B_{z}<0$ and $B_{y}<0$.

As discussed by Jackman et al. (2004) the compression regions (for which our fast flow model is relevant) last for a few days at a time, such that the more extreme cases of the cusp emissions may only be present for a few days during each solar rotation (typically $\sim 8$ days out of $\sim 25$ ). Jackman et al. (2004) note that significant north-south fluctuations are present on time scales of a few tens of minutes to a few hours during such intervals, and as such we might expect that the cusp related emissions to last typically of the same length. Interestingly, for the Northern Hemisphere our conceptual model also suggests that the cusp emissions relating to northward IMF and sub-solar reconnection will be stronger for negative IMF $B_{y}$. The same is true for the case of high-latitude lobe reconnection during intervals of southward IMF and positive IMF $B_{y}$. In the Southern Hemisphere, the flows associated with IMF $B_{y}$ are reversed, and hence emissions during intervals of northward or southward IMF will be brightest for positive or negative IMF $B_{y}$ respectively. Overall our model of pulsed reconnection under conditions of northwards and southwards IMF, and for varying orientations of IMF $B_{y}$, is found to produce a range of UV emission intensities and geometries which is in good agreement with the data presented by Gérard et al. (2004). However, we have no information on the prevailing solar wind and IMF structure during the interval discussed by Gérard et al. (2004). Of course, with the recent HST-Cassini solar wind campaign which took place in January 2004, we can now directly test the theoretical ideas presented here.

\section{Appendix A}

Here we describe, in detail, the parameters which are shown in Tables $2 \mathrm{a}$ and $\mathrm{b}$, and indicate how they were derived. From row (b) onwards parameters are shown first for the case of $B_{y}=0$ followed by the numbers shown in parentheses which indicate $B_{y}>0$ and $B_{y}<0$ conditions respectively. From the top, the rows of the table give information as follows:

1. The region in the model corresponding to the main upward field-aligned currents produced by the cusp flow pulses, together with its area $\left(\mathrm{m}^{2}\right)$.

2. The maximum magnitude of the field-aligned current density within the area given by (a), having (in the model) the same value on either side of the open-closed field line boundary.

3. The magnitude of the field-aligned current density averaged over the area given in (a), obtained from the total current in (d) divided by the area in (a).

4. The total upward-directed field-aligned current integrated over the area defined in (a). (Any small region of downward current in the area is not included.)

5. The limiting current density that can be carried in each region without field-aligned acceleration, given by Eq. (10). These values are also given in row (c) of Table 1 .

6. The maximum value of $r_{\min }$, the minimum kronocentric radial distance of the "top" of the particle acceleration region, obtained using Eq. (13) from the maximum current density shown in row (b) and the limiting current density for no field-aligned acceleration in row (e).

7. The "average" value of $r_{\text {min }}$, obtained as in row (f) but using the averaged field-aligned current density given in row (c).

8. The maximum value of the field-aligned voltage corresponding to the maximum required current density given in row (b), given by Eq. (12).

9. The "average" field-aligned voltage, defined by $\left\langle\Phi_{\|}\right\rangle=\int d A j_{\| i} \Phi_{\| i} / I_{||}$, such that the total electromagnetic energy input to the given region of ionosphere is equal to this "averaged" voltage times the total fieldaligned current. 
10. The maximum value of the precipitating particle energy flux $E_{f}$ corresponding to the maximum value of the field-aligned current density given in row (b), given by Eq. (14).

11. The value of the precipitating particle energy flux averaged over the area in row (a), obtained from the total power integrated over the area given in row (1), divided by the area in row (a).

12. The total precipitating particle power obtained by integrating $E_{f}$ over the areas given in row (a).

13. The maximum UV emission intensity, obtained from the maximum precipitating energy flux shown in row (j), assuming $\sim 10 \%$ of the energy flux is emitted as $10 \mathrm{eV}$ photons.

14. The averaged UV intensity, obtained as in row (m), but from the averaged precipitating energy flux shown in row $(\mathrm{k})$.

15. The total UV power emitted from the area given in (a), obtained as $20 \%$ of the total precipitating particle power in row (1).

Acknowledgements. EJB was supported during the course of this study by PPARC Postdoctoral Fellowship PPA/P/S/2002/00168. SWHC and SEM are supported by PPARC grant no. PPA/N/S/2000/00197. EJB would like to thank J.-C. Gérard, D. Grodent, and T. Yeoman for useful discussions.

The Editor in chief thanks a referee for his/her help in evaluating this paper.

\section{References}

Broadfoot, A. L., Sandel, B. R., Shemansky D. E., Holberg, J. B., Smith, G. R., Strobel, D. F., McConnell, J. C., Kumar, S., Hunten, D. M., Atreya, S. K., Donahue, T. M., Moos, H. W., Bertaux, J. L., Blamont, J. E., Pomphrey, R. B., and Linik, S.: Extreme ultraviolet observations from Voyager 1 encounter with Saturn, Science, 212, 206-208, 1981.

Bunce, E. J. and Cowley S. W. H.: Divergence of the equatorial current in the dawn sector of Jupiter's magnetosphere: analysis of Pioneer and Voyager magnetic field data, Planet. Space Sci., 49, 1089-1109, 2001.

Bunce, E. J., Cowley, S. W. H., and Wild, J. A.: Azimuthal magnetic fields in Saturn's magnetosphere: Effects associated with plasma sub-corotation and the magnetopause-tail current system, Ann. Geophys., 21, 1709-1724, 2003,

SRef-ID: 1432-0576/ag/2003-21-1709.

Bunce, E. J., Cowley, S. W. H., and Yeoman, T. K.: Jovian cusp processes: Implications for the polar aurora, J. Geophys. Res., 109, doi:10.1029/2003JA010280, 2004.

Burke, W. J., Kelley, M. C., Sagalyn, R. C., Smiddy, M., and Lai, S. J.: Polar cap electric field structures with a northward interplanetary magnetic field, Geophys. Res. Lett., 6, 21-25, 1979.

Clarke, J.T., Moos, H.W., Atreya, S.K., and Lane, A.L.: IUE detection of bursts of H Ly $\alpha$ emission from Saturn, Nature, 290, 226-230, 1981.
Clarke, J. T., Gérard, J.-C., Grodent, D., Wannawichian, S., Gustin, J., Connerney, J., Crary, F., Dougherty, M., Kurth, W., Cowley, S. W. H., Bunce, E. J., Hill, T., and Kim, J.: Morphological differences between Saturn's ultraviolet aurorae and those of Earth and Jupiter, Nature, 433, 717, 2005.

Cowley, S. W. H: Magnetospheric asymmetries associated with the y-component of the IMF, Planet. Space Sci., 29, 79-99, 1981.

Cowley, S. W. H. and Lockwood, M.: Excitation and decay of solar wind-driven flows in the magnetosphere-ionosphere system, Ann. Geophys., 10, 103-125, 1992.

Cowley, S. W. H., and Bunce, E. J.: Origin of the main auroral oval in Jupiter's coupled magnetosphere-ionosphere system, Planet. Space Sci., 49, 1067-1088, 2001.

Cowley, S. W. H., and Bunce, E. J.: Corotation-driven magnetosphere-ionosphere coupling currents in Saturn's magnetosphere and their relation to the auroras, Ann. Geophys., 21, 1691-1713, 2003, SRef-ID: 1432-0576/ag/2003-21-1691.

Cowley, S. W. H., Bunce, E. J., and Prangé, R.: Saturn's polar ionospheric flows and their relation to the main auroral oval, Ann. Geophys., 22, 1379-1392, 2004,

SRef-ID: 1432-0576/ag/2004-22-1379.

Cowley, S. W. H., Badman, S. V., Bunce, E. J., Jackman, C. M., Milan, S. E., and Yeoman, T. K.: Reconnection in a rotation-dominated magnetosphere and its relation to Saturn's auroral dynamics, J. Geophys. Res., 110(A02201), doi:10.1029/2004JA010796, 2005.

Cummings, W. D., O’Sullivan, R. J., and Coleman P. J.: Standing Alfvén waves in the magnetosphere, J. Geophys. Res., 74, 778$781,1969$.

Dungey, J. W.: Interplanetary field and the auroral zones, Phys. Rev. Lett., 6, 47-49, 1961.

Farrugia, C. J., Sandholt, P. E., Cowley, S. W. H., Southwood, D. J., Egeland, A., Stauning, P., Lepping, R. P., Lazarus, A. J., Hansen, T., and Friis-Christensen, E.: Reconnection-associated auroral activity stimulated by two types of upstream dynamic pressure variations: Interplanetary magnetic field conditions $\mathrm{B}_{z} \sim 0, \mathrm{~B}_{y} \ll 0$ case, J. Geophys. Res., 100, 21 753-21 764, 1995

Gérard, J.-C., Dols, V., Grodent, D., Waite, J. H., Gladstone, G. R., and Prangé, R.: Simultaneous observations of the saturnian aurora and polar haze with the HST/FOC, Geophys. Res. Lett., 22, 2685-2689, 1995.

Gérard, J.-C., Grodent, D., Gustin, J., Saglam, A., Clarke, J. T., and Trauger, J. T.: Characterisitics of Saturn's FUV aurora observed with the Space Telescope Imaging Spectrograph, J. Geophys. Res., 109, doi://10.1029/2004JA010513, 2004.

Gladstone, G. R., Waite, J. H. , Jr., Grodent, D., Lewis, W. S. , Crary, F. J., Elsner, R. F., Weisskopf, M. C., Majeed, T., Jahn, J.M., Bhardwaj, A., Clarke, J. T., Young, D. T., Dougherty, M. K., Espinosa, S. A., and Cravens, T. E.: A pulsating auroral X-ray hot spot on Jupiter, Nature, 415, 1000-1004, 2002.

Grodent, D., Clarke, J. T., Waite, J. H., Jr., Cowley, S. W. H., Gérard, J.-C., and Kim, J.: Jupiter's polar auroral emissions, J. Geophys. Res., 108(A10), 1366, doi10:1029/2003JA010017, 2003.

Heelis, R. A., Reiff, P. H., Winningham, J. D., and Hanson, W. B.: Ionospheric convection observed by DE 2 during northward interplanetary magnetic field, J. Geophys. Res., 91, 5817-5826, 1986.

Hill, T. W.: Inertial limit on corotation, J. Geophys. Res., 84, 6554 6565, 1979.

Hill, T. W.: The jovian auroral oval, J. Geophys. Res., 106, 8101- 
$8110,2001$.

Jackman, C. M., Achilleos, N., Bunce, E. J., Cowley, S. W. H., Dougherty, M. K., Jones, G. H., Milan, S. E., and Smith, E. J.: Interplanetary magnetic field at $\sim 9$ AU during the declining phase of the solar cycle and its implications for Saturn's magnetospheric dynamics, J. Geophys. Res., 109(A11203), doi:10.1029/2004JA010614, 2004.

Jørgensen, T. S., Friis-Christensen, E., and Wilhjelm, J.: Interplanetary magnetic field direction and high-latitude ionospheric currents, J. Geophys. Res., 77, 1976-1984, 1972.

Judge, D. L., Wu, F. M., and Carlson, R. W.: Ultraviolet photometer observations of the Saturnian system, Science, 207, 431-435, 1980.

Knight, S.: Parallel electric fields, Planet. Space Sci., 21, 741-751, 1973.

Lockwood, M., Cowley, S. W. H., and Freeman, M. P.: The excitation of plasma convection in the high-latitude ionosphere, J. Geophys. Res., 95, 7961-7975, 1990.

Lundin, R. and Sandahl, I.: Some characteristics of the parallel electric field acceleration of electrons over discrete auroral arcs as observed from two rocket flights, in: Symposium on European Rocket Research, ESA SP-135, ESA, Noordwijk, The Netherlands, p. 125, 1978.

Maezawa, K.: Magnetospheric convection induced by positive and negative $\mathrm{z}$ component of the interplanetary magnetic field: Quantitative analysis using polar cap magnetic records, J. Geophys. Res., 81, 2289-2301, 1976.

McWilliams, K. A., Yeoman, T. K., and Provan, G.: A statistical study of dayside pulsed ionospheric flows as seen by the CUTLASS Finland HF radar, Ann. Geophys., 18, 445-460, 2000,

SRef-ID: 1432-0576/ag/2000-18-445.

Milan, S. E., Lester, M., Cowley, S. W. H., and Brittnacher, M.: Dayside convection and auroral morphology during an interval of northward interplanetary magnetic field, Ann. Geophys., 18, 436-447, 2000,

SRef-ID: 1432-0576/ag/2000-18-436.

Milan, S. E., Cowley, S. W. H., Lester, M., Wright, D. M., Slavin, J. A., Fillingim, M., Carlson, C. W., and Singer, H. J.: Response of the magnetotail to changes in the open flux content of the magnetosphere, J. Geophys. Res., 109(A04220), doi:10.1029/2003JA010350, 2004.

Milan, S. E.: A simple model of the flux content of the distant magnetotail, J. Geophys. Res., 109(A07210), doi:10.1029/2004JA010397, 2004.

Milan, S. E., Hubert, B., and Grocott, A.: Formation and motion of a transpolar arc in response to dayside and nightside reconnection, J. Geophys. Res., 110(A01212, doi:10.1029/2004JA010835, 2005.

Metzger, A. E., Gilman, D. A., Luthey, J. L., Hurley, K. C., Schnopper, H. W., Seward, F. D., and Sullivan, J. D.: The detection of X-rays from Jupiter, J. Geophys. Res., 88, 7731-7742, 1983.

Neudegg, D. A., Yeoman, T. K., Cowley, S. W. H., Provan, G., Haerendel, G., Baumjohann, W., Auster, U., Fornacon, K.-H., Georgescu, E., and Owen, C. J.: A flux transfer event observed at the magnetopause by the Equator-S spacecraft and in the ionosphere by the CUTLASS HF radar, Ann. Geophys., 17, 707-723, 1999 ,

SRef-ID: 1432-0576/ag/1999-17-707.

Øieroset, M., Sandholt, P. E., Denig, W. F., and Cowley, S. W. H.: Northward interplanetary magnetic field cusp aurora and high-latitude magnetopause reconnection, J. Geophys. Res., 102, 11349-11360, 1997.
Pallier, L. and Prangé, R.: More about the structure of the high latitude jovian aurorae, Planet. Space Sci., 49, 1159-1173, 2001.

Pallier, L. and Prangé, R.: Detection of the southern counterpart of the northern polar cusp: Shared properties, Geophys. Res. Lett., 31 Eq. (6), Art. No. LO6701, 2004.

Paschmann, G., Haaland, S., and Treumann, R.: Auroral plasma physics, Space Sci. Rev., 103, 475, 2002.

Phan, T., Frey, H. U., Frey, S., Peticolas, L., Fuselier, S., Carlson, C., Rème, H., Bosqued, J.-M., Balogh, A., Dunlop, M., Kistler, L., Mouikis, C., Dandouras, I., Sauvaud, J.-A., Mende, S., McFadden, J., Parks, G., Moebius, E., Klecker, B., Paschmann, G., Fujimoto, M., Petrinec, S., Marcucci, M. F., Korth, A., and Lundin, R.: Simultaneous Cluster and IMAGE observations of cusp reconnection and auroral proton spot for northward IMF, Geophys. Res. Lett., 30(10), 1509-1513, doi:10.1029/2003GL016885, 2003.

Provan, G., Yeoman, T. K., and Milan, S. E.: CUTLASS Finland radar observations of the ionospheric signatures of flux transfer events and the resulting plasma flows, Ann. Geophys., 16, 14111422, 1998,

\section{SRef-ID: 1432-0576/ag/1998-16-1411.}

Reiff, P. H., Burch, J. L., and Spiro, R. W.: Cusp proton signatures and the interplanetary magnetic field, J. Geophys. Res., 85, 5997-6010, 1980.

Richardson, J. D., Thermal ions at Saturn: Plasma parameters and implications, J. Geophys. Res., 91, 1381-1392, 1986.

Richardson, J. D.: A new model for plasma transport and chemistry at Saturn, J. Geophys. Res., 97, 13 705-13 716, 1992.

Richardson, J. D.: An extended plasma model for Saturn, Geophys. Res. Lett., 22, 1177-1181, 1995.

Richardson, J. D. and Sittler, E. C., Jr.: A plasma density model for Saturn based on Voyager observations, J. Geophys. Res., 95, 12 019-12 028, 1990.

Richardson, J. D., Eviatar, A., McGrath, M. A., and Vasyliunas, V. M.: OH in Saturn's magnetosphere: Observations and theory, J. Geophys. Res., 103, 20 245-20 250, 1998.

Russell, C. T., and Elphic, R. C.: Initial ISEE magnetometer results: magnetopause observations, Space Sci. Rev., 22, 681-695, 1978.

Sandel, B. R. and Broadfoot, A. L.: Morphology of Saturn's aurora, Nature, 292, 679-685, 1981.

Sandel, B. R., Shemansky, D. E., Broadfoot, A. L., Holberg, J. B., Smith, G. R., McConnell, J. C., Strobel, D. F., Atreya, S. K., Donahue, T. M., Moos, H. W., Hunten, D. M., Pomphrey, R. B., and Linik, S.: Extreme ultraviolet observations from the Voyager 2 encounter with Saturn, Science, 215, 548-553, 1982.

Sandholt, P. E., Farrugia, C. J., Moen, J., and Cowley, S. W. H.: Dayside auroral configurations: Responses to southward and northward rotations of the interplanetary magnetic field, J. Geophys. Res. Vol. 103(No.A9), 20-31, 279(98JA01541), 1998.

Saur, J., Mauk, B. H., Kaßner, A., and Neubauer, F. M.: A model for the azimuthal plasma velocity in Saturn's magnetosphere, J. Geophys. Res., 109(A05217), doi:10.1029/2003JA010207, 2004.

Sckopke, N. and Paschmann, G.: The plasma mantle: A survey of magnetotail boundary layer observations, J. Atmos. And Terrestrial Phys., 40, 261-278, 1978.

Shemansky, D. E. and Ajello, J. M.: The Saturn spectrum in the EUV: Electron excited hydrogen, J. Geophys. Res., 88, 459-471, 1983.

Siscoe, G. L. and Huang, T. S.: Polar cap inflation and deflation, J. Geophys. Res., 90, 543-554, 1985.

Sittler, E. C., Jr., Olgivie, K. W., and Scudder, J. D.: Survey of lowenergy plasma electrons in Saturn's magnetosphere: Voyagers 1 
and 2, J. Geophys. Res., 88, 8847-8858, 1983.

Spreiter, J. R., Summers, A. L., and Alksne, A. Y.: Hydromagnetic flow around the magnetosphere, Planet. Space Sci., 14, 223-237, 1966.

Southwood, D. J and Kivelson, M. G.: A new perspective concerning the influence of the solar wind on the jovian magnetosphere, J. Geophys. Res., 106, 6123-6132, 2001.

Stallard, T. S., Miller, S., Trafton, L. M., Geballe, T. R., and Joseph, R. D.: Ion winds in Saturn's southern auroral/polar region, Icarus, 167, 204-215, 2004.

Taylor J. R., Cowley, S. W. H., Yeoman, T. K., Lester, M., Jones, T. B., Greenwald, R. A., Sofko, G., Villain, J.-P., Lepping, R. P., and Hairston, M. R.: SuperDARN studies of the ionospheric convection response to a northward turning of the interplanetary magnetic field, Ann. Geophys., 16, 549-562, 1998,

SRef-ID: 1432-0576/ag/1998-16-549

Trauger, J. T., Clarke, J. T., Ballester, G. E., Evans, R. W., Burrows, C. J., Crisp, D., Gallagher, J. S., III, Griffiths, R. E., Hester, J. J., Hoessel, J. G., Holtzman, J. A., Krist, J. E., Mould, J. R., Sahai, R., Scowen, P. A., Stapelfeldt, K. R., and Watson, A. M.: Saturn's hydrogen aurora: Wide field and planetary camera 2 imaging from the Hubble Space Telescope, J. Geophys. Res., 103, 20 237-20 248, 1998.
Vasyliunas, V. M.: Plasma distribution and flow, in Physics of the Jovian Magnetosphere, edited by A.J. Dessler, p. 395, Cambridge Univ. Press, Cambridge, UK, 1983.

Waite, J. H., Jr., Bagenal, F., Seward, F., Na, C., Gladstone, G. R., Cravens, T. E., Hurley, K. C., Clarke, J. T., Elsner, R., and Stern, S. A.: ROSAT observations of the Jupiter aurora, J. Geophys. Res., 99, 14 799-14 810, 1994.

Waite, J. H., Jr., Gladstone, G. R., Lewis, W. S., Goldstein, R., McComas, D. J., Riley, P., Walker, R. J., Robertson, P., Desai, S., Clarke, J. T., and Young, D. T. : An auroral flare at Jupiter, Nature, 410, 787-791, 2001.

Wild, J. A., Cowley, S. W. H., Davies, J. A., Khan, H., Lester, M., Milan, S. E., Provan, G., Yeoman, T. K., Balogh, A., Dunlop, M. W., Fornacon, K.-H., and Georgescu, E.: First simultaneous observations of flux transfer events at the high-latitude magnetopause by the Cluster spacecraft and pulsed radar signatures in the conjugate ionosphere by the CUTLASS and EISCAT radars, Ann. Geophys., 19, 1491-1505, 2001,

SRef-ID: 1432-0576/ag/2001-19-1491. 